\title{
Méthode graphique
}

\section{pour le calcul des cheminées d'équilibre}

\section{Graphical method for calculating surge-tanks}

\author{
PAR 1I. BOUVARD ET J. MOLBER'T \\ IxGÉNEUTS A L'ELECrRICITE DE FRANCE \\ R.E.H. Alpes II
}

English synopsis, p. 527.

\section{I. - PRÉlIMINAIRES}

\section{A) Sources}

La méthode que nous proposons nous a été suggérée par un article du Docteur-Ingénieur Traugott Schirfmann, publić dans Wasserkraft und Wasserwirtschaft de mars 1942.

Cette méthode avait dejà été traduite et complétée par le Docteur-Ingénieur Del Felice dans un article paru dans Energia Elettrica du 4 mai 1947.

Le principe fondamental restant le même, nous avons repris et modifié la méthode de Schrr:MANN quil laissait place à une erreur systématique que nous pensons avoir pratiquement annulée.

\section{B) Plan suivi}

Nous allons, dans cette étude :

$1^{\circ}$ Exposer le principe de la mithode graphique en insistant, en particulier, sur la notion de hauteur accélératrice;

2" Appliquer ces principes à la construction d'épures permettant d'étudier une cheminée d'équilibre d'un type quelconque au cours de manouvres d'ouvertures ou de fermetures totales ou partielles, instantanées ou non;

3" Donner en exemples quatre épures relatives à :

- une cheminée à expansion,

- une cheminée à étranglement,

-_ une cheminée déversante,

- une cheminée difrérentielle;
4. Etendre la construction graphique au cas plus complique àune cheminée d'équilibre branchée sur une galerie en $Y$, les deux prises d'eau clant à des niveaux statiques différents.

- Etudier rapidement le cas particulier d'une galerie en $V$.

- Illustrer par deux épures cette dernière partie.

\section{II. - PRINCIPE DE LA MÉTHODE}

\section{A) Notion de hauteur accélératrice $H_{a}$}

Nous supposons connue la base thérique des cheminées d'équilibre.

Considérons le système prise d'eau - cheminće d'équilibre - conduite forcée, représenté par la figure 1.

Désignons par :

F la section de la cheminée d'équilibre;

$f$ la section de la galerie:

I. Ia longueur de la galerie d'amenée;

$Z_{0}$ la cote de l'eau à la prise d'eau;

$Z_{v}$ la cote de l'eau dans la cheminée d'équilibre en régime variable;

$Z_{p}$ la cote de l'eau dans la cheminée d'équilibre en régime permanent fictif (défini uîtérieurement).

Les débits de la galerie, de la cheminée d'équilibre el de la conduite forcée sont respectivement $Q_{1}, Q_{2}, Q_{3}$; nous conviendrons quils seront représentés par des nombres positifs lorsque le 
courant se dirigera vers le point $M$, et négatifs dans le cas contraire. Moyennant cette convention, on pourra écrire à chaque instant :

$$
Q_{1}+Q_{2}+Q_{3}=0 .
$$

$\mathrm{R}_{1}$ et $\mathrm{R}_{2 t}$ dans la galerie et dans l'étranglement, soit :

$$
Z_{0}+\mathrm{R}_{1 t}+\mathrm{R}_{2 t}=Z_{p t}
$$

En régime varié, nous appellerons hauteur ac-

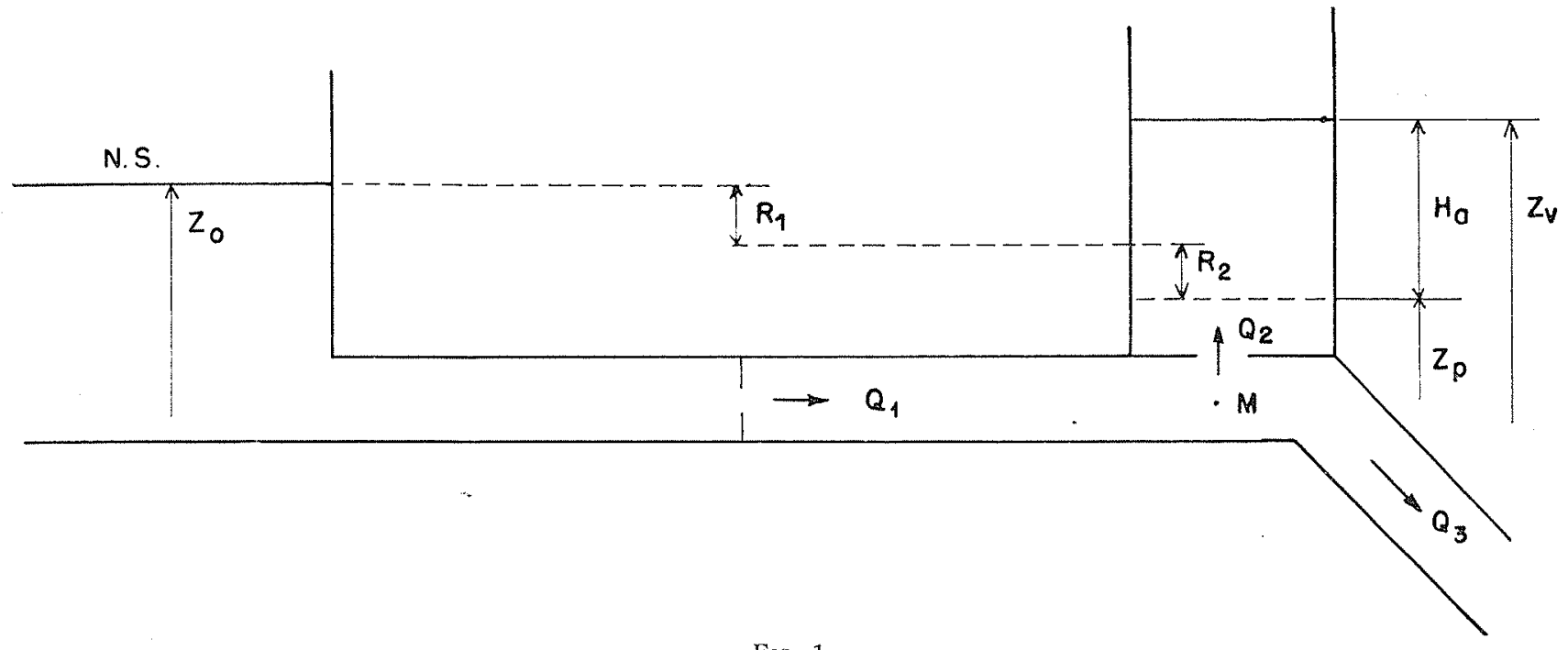

FIG. 1.

Les pertes de charge $R_{I}$ dans la galerie seront supposées, pour simplifier, concentrées en un diaphragme unique. Elles sont de la forme :

$$
\mathrm{R}_{1}=\mathrm{K}_{1}\left(\mathrm{Q}_{1}\right)^{2} \text {. }
$$

De même les pertes de charge du diaphragme de la cheminée d'équilibre sont :

$$
\mathrm{R}_{2}=\mathrm{K}^{2}\left(\mathrm{Q}_{2}\right)^{2} \text {. }
$$

$Q_{1}$ et $Q_{2}$ sont représentés par des nombres algébriques. Il en est de mème de $R_{1}$ et $R_{2}$ avec les conventions nécessaires.

Néanmoins, dans ce chapitre, nous ne nous étendrons pas sur les questions de signe, qui risqueraient d'alourdir ce bref exposé.

En régime variable, à un instant $t$ donnć, les débits passant dans la galerie, la cheminée d'équilibre et la conduite forcée sont respectivement $Q_{1 t}-Q_{2 t}-Q_{3 t}: Q_{3}$ est constant, $Q_{1}$ et $Q_{2}$ varient, (nous verrons ultérieurement comment ramener au cas $Q_{3}$ constant le cas $Q_{3}$ variable).

Supposons qu'il existe un régime permanent caractérisé par les débits $\mathrm{Q}_{1 t}-\mathrm{Q}_{2 i}-\mathrm{Q}_{3 t}$ (il suffit d'imaginer, par exemple, un déversoir placé à une cote judicieuse dans la cheminée d'équilibre permettant au débit $Q_{2 t}$ de rentrer dans celle-ci sans que le niveau de l'cau ne varie); le niveau de l'eau dans la cheminée d'équilibre se déduirait du N.S. de $\operatorname{cote} Z_{0} \dot{a}$ la prise d'eau en ajoutant à $Z_{0}$ (algébriquement) les pertes de charge célératrice $\mathrm{H}_{a}$ la différence $Z_{v}-Z_{p}$ entre le niveau de l'eau $Z_{v}$ dans la cheminée d'équilibre a l'instant $t$ et le niveau fictif $Z_{p}$ que nous venons de définir. Cette hauteur $H_{a}$ représente une charge qui a tendance à faire varier le régime de l'eau dans la galerie.

$$
\begin{gathered}
\mathrm{H}_{a} \text { ser } \mathbf{a}>0 \text { ou }<0 \\
\text { suivant } \text { que } Z_{v} \text { sera }>\text { ou }<Z_{p} .
\end{gathered}
$$

L'accélération de la masse d'eau contenue dans la galerie d'amenée étant proportionnelle à la force $f \mathrm{H}_{a}$ qui s'exerce sur elie, on peut écrire :

$$
f \mathrm{H}_{a}=\frac{\mathrm{L}}{g} f \frac{d \mathrm{~W}}{d t}
$$

(W étant la vitesse de l'eau dans la galerie), ce qui est une interprétation direcle de la formule classique :

$$
F=m \%
$$

Nous retrouvons ainsi, par un raisonnement un peu intuitif, les équations fondamentales des cheminées d'équilibre.

Si nous remarquons que :

$$
\begin{aligned}
\mathrm{W} & =\frac{\mathrm{Q}_{1}}{f} \\
d \mathrm{~W} & =\frac{d \mathrm{Q}_{1}}{f}
\end{aligned}
$$


On en déduit :

$$
\frac{\mathrm{L}}{g} \frac{d \mathrm{Q}_{1}}{d t}=f \mathrm{H}_{t}
$$

d'où :

$$
d \mathrm{Q}_{1}=-\frac{g f}{\mathrm{I}} \mathrm{H}_{a} d t=-d \mathrm{Q}_{2}
$$

En remarquant que de l'expression :

$$
Q_{1}+Q_{2}+Q_{3}=0
$$

on tire :

$$
d \mathrm{Q}_{1}+d \mathrm{Q}_{2}=0 \quad\left(\mathrm{Q}_{3}=\mathrm{C}^{\mathrm{te}}\right)
$$

Passant des différentielles à des valeurs petites mais finies des variables, nous écrirons :

$$
\Delta Q=\Delta Q_{1}=-\Delta Q_{2}=\frac{g f}{\mathrm{~L}}-\mathrm{H}_{n} \Delta t
$$

ou encore :

$$
\Delta Q=\alpha \mathbf{H}_{a} \Delta t \quad \text { en posant } \alpha=\frac{g}{\text { L. }} f
$$

ce que nous interpréterons et résumerons ainsi :

La variation de débil dans la galerie (ou dans la cheminée d'équilibre puisque $Q_{3}=$ constante) est proportionnelle au temps et à la hauteur accélératrice $\mathrm{H}_{a}$.

$\mathrm{H}_{a}$ représentant à an instant donné la diffirence entre le nivean réel dans la chemine d'équilibre et le nivean fictif qui existerait dans la cheminée d'équilibre si le régime dtail permanent (les débits étant répartis comme les débits instantanés au temps $t \mathrm{Q}_{1}, \mathrm{Q}_{2}, \mathrm{Q}_{3 t}$ ).

\section{B) Utilisation de cette notion}

a) DFfinition dF l'état de système au TEmps $t$ :

Supposons connus au temps $t, Q_{11}$, $Q_{3 t}$ et $Z_{11}$, nous pourrons en déduire toutes les autres variables du système et, en particulier :

-. Le débit entrant dans la cheminée d'écuilibre.

$$
\mathrm{Q}_{2 t}=-\cdots\left(\mathrm{Q}_{1 t}+\mathrm{Q}_{3 t}\right)
$$

-- Ies pertes de charge :

$$
\begin{aligned}
& \mathrm{R}_{1 t}=\mathrm{K}_{1}\left(\mathrm{Q}_{1 t}\right)^{2} \\
& \mathrm{R}_{2 t}=\mathrm{K}_{2}\left(\mathrm{Q}_{2 i}\right)^{2}
\end{aligned}
$$

- Puis, en donnant à $R_{1 t}$ et $R_{2, t}$ des signes adéquats, liés aux directions de l'écoulement :

$$
Z_{p t}=Z_{0}+\mathrm{R}_{1 t}+\mathrm{R}_{2 t}
$$

d'où :

$$
\mathbf{H}_{a t}=Z_{t t}-Z_{p t}
$$

\section{Conctusion}

Connaissant $\mathrm{Q}_{1 t}, \mathrm{Q}_{3 !}$ et $Z_{t}$, en peut calculer $Q_{2 i} \in t \mathrm{H}_{u t}$.

b) Etat du systeme au temps $t+\Delta t$.

Considérons un intervalle de temps $\Delta t$ suffisamment petit pour qu'on puisse considérer que les variations de $Q_{2}$ et de $\mathrm{H}_{a}$ soient négligeables dans cet intervalle; on pourra déduire de ces valeurs supposées fixes l'état du système à l'instant $t+\Delta t$.

En effet, nous pouvons calculer :

- la variation de débit $\Delta Q$ :

$$
\Delta \mathrm{Q}=\alpha_{\mathrm{H}} \mathrm{H}_{t t} \Delta t
$$

d'où :

$$
\mathrm{Q}_{(t+\Delta t)}=Q_{2 t}+\Delta \mathrm{Q}
$$

- la montée ou la descente de l'eau dans la cheminée d'équilibre : $\Delta Z$ :

$$
\Delta Z=\frac{Q_{2 t}}{\mathrm{~F}} \Delta t
$$

d'où :

$$
Z_{0(t+\Delta t)}=Z_{t,}+\Delta Z
$$

Le débit $Q_{3}$ n'ayant pas changé, nous connaissons l'état du système à l'instant $t+\Delta t$.

\section{c) Représfintation gRaphique.}

La méthode que nous exposons n'est pas autre chose que l'interprétation graphique de ce résultat.

Nous considérerons un système d'axes de coordonnces rectangulaires, dans lequel nous porterons en abscisses les débits $Q_{1}$ dans la galerie et en ordonnées les niveaux de l'eau $Z_{v}$ dans la cheminée d'équilibre. Un mouvement non permanent du système sera représenté sur ce plan par une courbe $(\mathrm{C})$.

La construction graphique consistera à construire une ligne brisée $A B C$ inscrite dans cette courbe théorique et qui sera formée d'une succession de petits segments représentant les variations supposées linćaires du phénomène durant des intervalles de temps petits $\Delta t$ (fig. 2 ). 


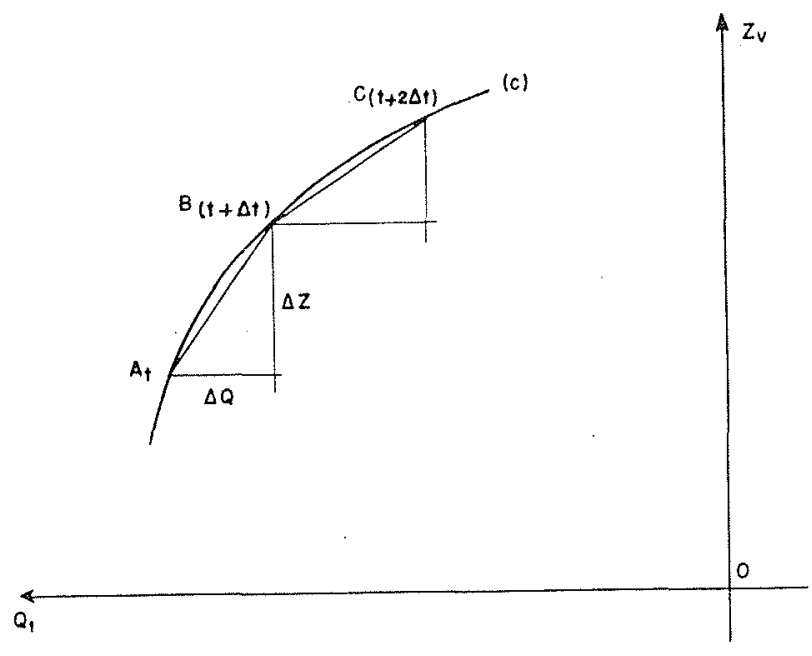

FII, 2.

A partir de At déjà trouvé, on pourra construire le point $B_{(t+\Delta t)}$ en calculant séparément $\Delta Q$ et $\Delta Z$, etc...

\section{III. - CONSTRUCTION DE L'ÉPURE}

\section{A) Disposition de l'épure}

Au centre de notre épure (fig. 3), nous tracons les axes de coordonnées $\mathrm{QQ}^{\prime}$ et $Z Z^{\prime}$ : débit dans la galerie - niveau de l'eau dans la cheminée d'équilibre. Nous avons compté vers la gauche les $Q_{1}$ positifs. L'axe des abscisses est à la cote du niveau statique à la prise d'eau.

Nous traçons aussi la parabole des pertes de charge dans la galerie, en fonction du débit. Les points de cette parabole représentent le niveau piézométrique de l'eau au droit de la chemince d'équilibre en régime permanent.

Dans le bas de l'épure, nous tracons un deuxième axe des abscises $\left(O^{\prime} \gamma^{\prime}\right)$ sur lequel nous mesurons les débits $Q_{3}$ (en inversant le sens positif, les $Q_{3}$ négatifs étant dirigés vers la gauche). Ces débits sont fonction de l'ouverture des turbines et cet axe pourra aussi bien être gradué en ouverture.

'Traçons également l'axe $\alpha q^{\prime}$ coupant $\mathrm{O}^{\prime} y^{\prime}$ à la distance $Q_{3}$ du point $O$. Soit $A$ un point quelconque du plan; la distance de $\mathrm{A}$ à l'axe $\alpha \alpha^{\prime}$ mesurera le débit $Q_{2}$ entrant dans la cheminée d'équilibre (figure 4).

Nous terminerons la préparation de notre épure en traçant les courbes (1) - (2) - (3) - (4) et éventuellement (5) dont nous explique. rons plus Ioin l'utilité (fig. 3).

\section{B) Méthode de première approximation Construction du point $\mathrm{B}(t+\Delta t)$ à partir du point $A_{t}$}

Soit le point $A_{i}$ représentant l'état de la cheminée d'équilibre à l'instant $t$. Nous allons indiquer

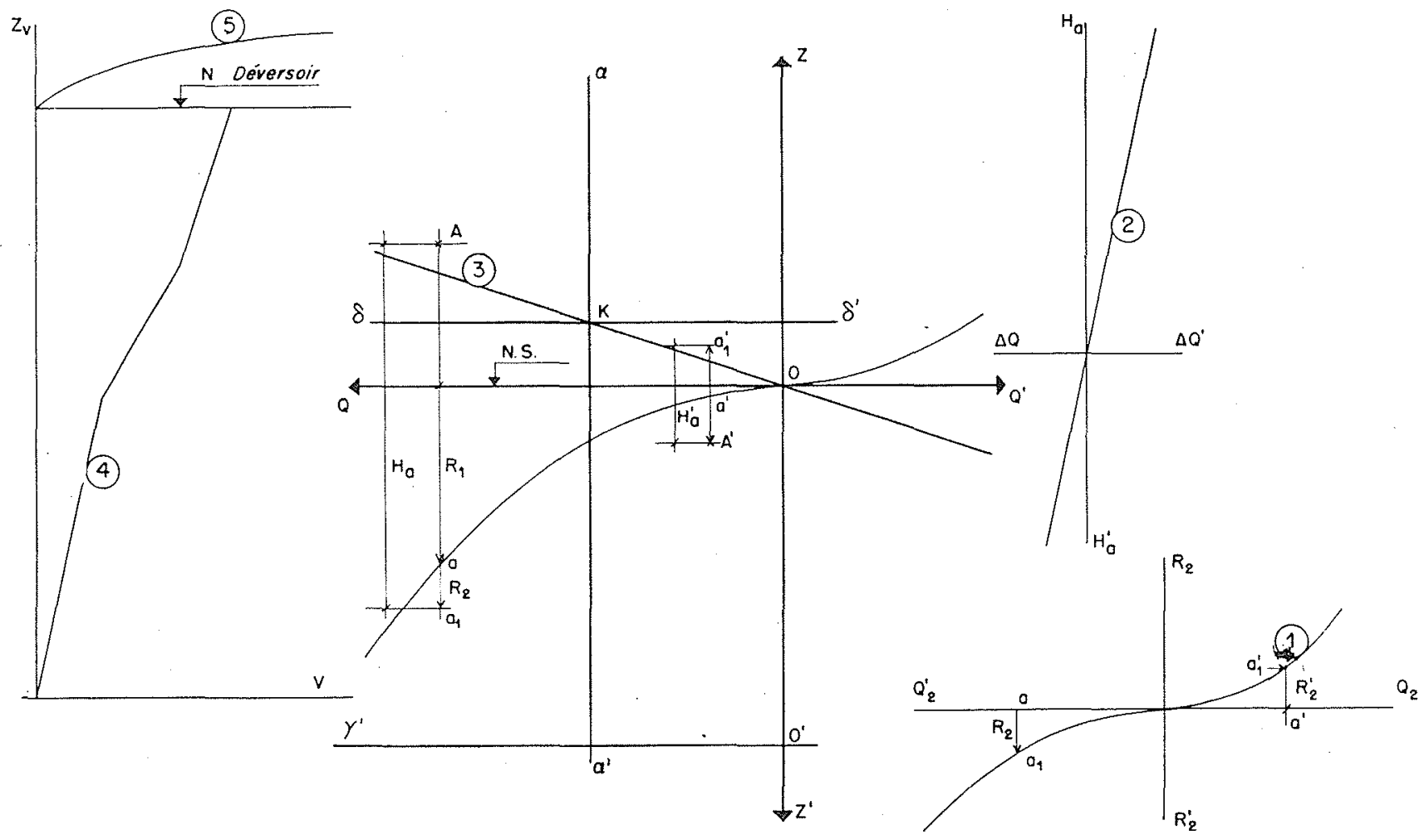

Fic. 3 . 
comment on peut construire le point $\mathrm{B}(t+-j t)$ (représentatil de l'état de la cheminée décquilibre au temps $t+\Delta t$ ) à patir du point $A_{1}$.

\section{(a) Determanatox de $\mathrm{H}_{\text {at }}$ (voll fig. 3 ).}

Tracons dans un coin de notre feuille la courbe (1) nous donnant les pertes de charge $R_{\text {. }}$ à travers l'étranglement, en fonction de $\mathrm{Q}$ (i) est commode de compter les Q négatifs vers la gauche et de tracer la courbe comme il est indique sur la figure 3 ).

Considérons le point A de la figure 3 , le point a de même abscisse situé dans la parabole des perles de charge représente le niveau piézométrique fictif en régime permanent sous la cheminée d'équilibre. Si, à partir de a comme origine, nous portons le vecteur $R_{2}$ donné par la courbe (1), nous obtiendrons le point $a_{1}$ dont la cote représentera le niveau fictif, en régime permanent, de l'eau dans la cheminée d'épulibre (niveau dont nous avons parlé au chapitre precedent) défni

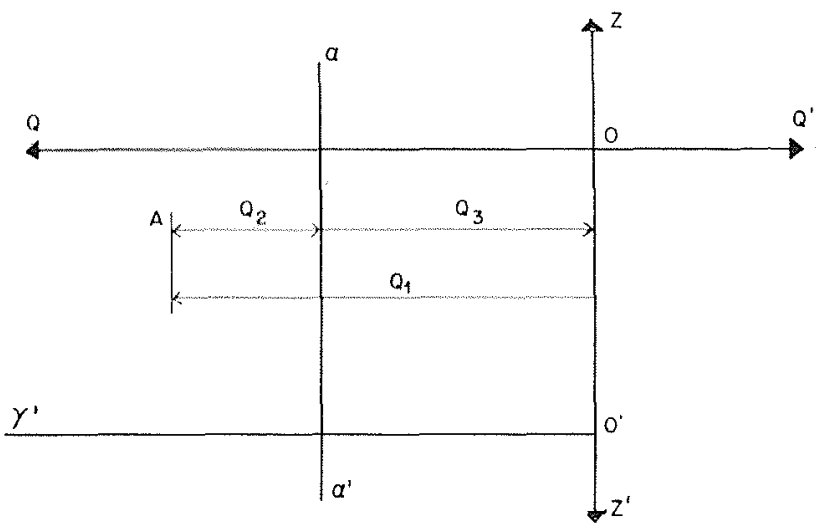

FIti. 4.

par les débits $Q_{1}-Q_{2}-Q_{n}$. La mesure de $A a_{1}$ nous donnera la hatuteur accélératrice $\mathrm{H}_{a}$.

Sur la figure 3, nous avons représenté, outre la construction de $H_{n}$ relative au point $A$, la même construction relative au point $A^{\prime}$ situé dans une autre partie du plan, ceci pour indiquer comment on doit se servir des vecteurs $R_{22}$ donnés par la courbe (1).

\section{b) DétrRmination de $\Delta Q[$ courbe $(2)]$.}

Dans un systeme daxe $\left(H_{a} ; \Delta Q\right)$ et pour un $\Delta t$ donné, on trace la droite (2) :

$$
\Delta \mathrm{Q}=\alpha \mathrm{H}_{a} \Delta t
$$

(Les unites sur ce graphique sont à la mème échelle que sur les axes $Q Q^{\prime}$ et $Z Z^{\prime}$ de l'épure.)

Comaissant $\mathrm{H}_{a}$, la droite (2) nous donnera le AQ correspondant, d'où l'abscisse du point $B$.
Dans lexemple de la figure $5, H_{a}$ est posilif, $\left(Z_{1}>Z_{p}\right)$ et $\Delta \mathrm{Q}$ negatif.

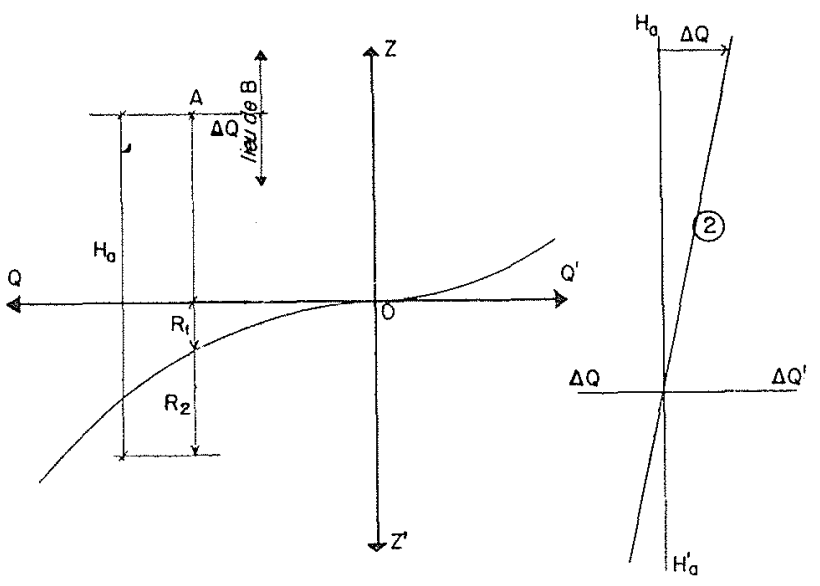

FIG. 5.

c) DETERMINATON Dr: $\triangle Z$ (COLRBES 3 RT 4 ).

Donnons-nous une echelle convenable des volumes et tracons :

1" La droite (3) d'equation $\Delta V=\mathrm{Q} \Delta t$.

Il est commode de prendre l'axe $Z Z^{\prime}$ pour axe des abscisses et de faire passer la droile (3) par le point $O$, comme il est indiqué sur la figure 6 .

2" Ia coube des volumes (4) donnant dans un syslome d'axe (volume-hauteur d'eau) le volume d'eau emmagasiné dans la cheminé d'equilibre en fonction du niveau.

L'axe as' coupe la droite (3) en K. Par K, menons lat parallè ồo à l'axe des abscisses. L'axe et la droite (3) découpent sur une verticale passant par A le segment $c d$ représentant le $\Delta V$ entrant dans ta cheminée d'écuilibre pendant le temps $\Delta t$.

La figure 6 montre mieux que tout commentaire la construction tres simple qui pernet d'oblenir l'ordonnee de $B_{(t+\Delta t)}$.

Remarque. - Pour diminuer les risques d'erreur dans les cas compliqués, on représente $c d$ par une fleche ayant son origine en $c$ sur l'axe $\hat{\delta} \hat{\delta}^{\prime}$ et son extrémité en $d$ sur la droite (3). Si cette flèche est dirigée vers le haut, l'eau monte dans la cheminée d'équilibre (exemple : point A), elle descend dans le cas contraire (exemple : point $\left.A^{\prime}\right)$.

d) Cheminté Avec Dléversork [courbe (5)].

On emploie la courbe (5) (fig. 3), qui domne, en fonction du débit déversé, le niveau de l'eau au-dessus du déversoir. 


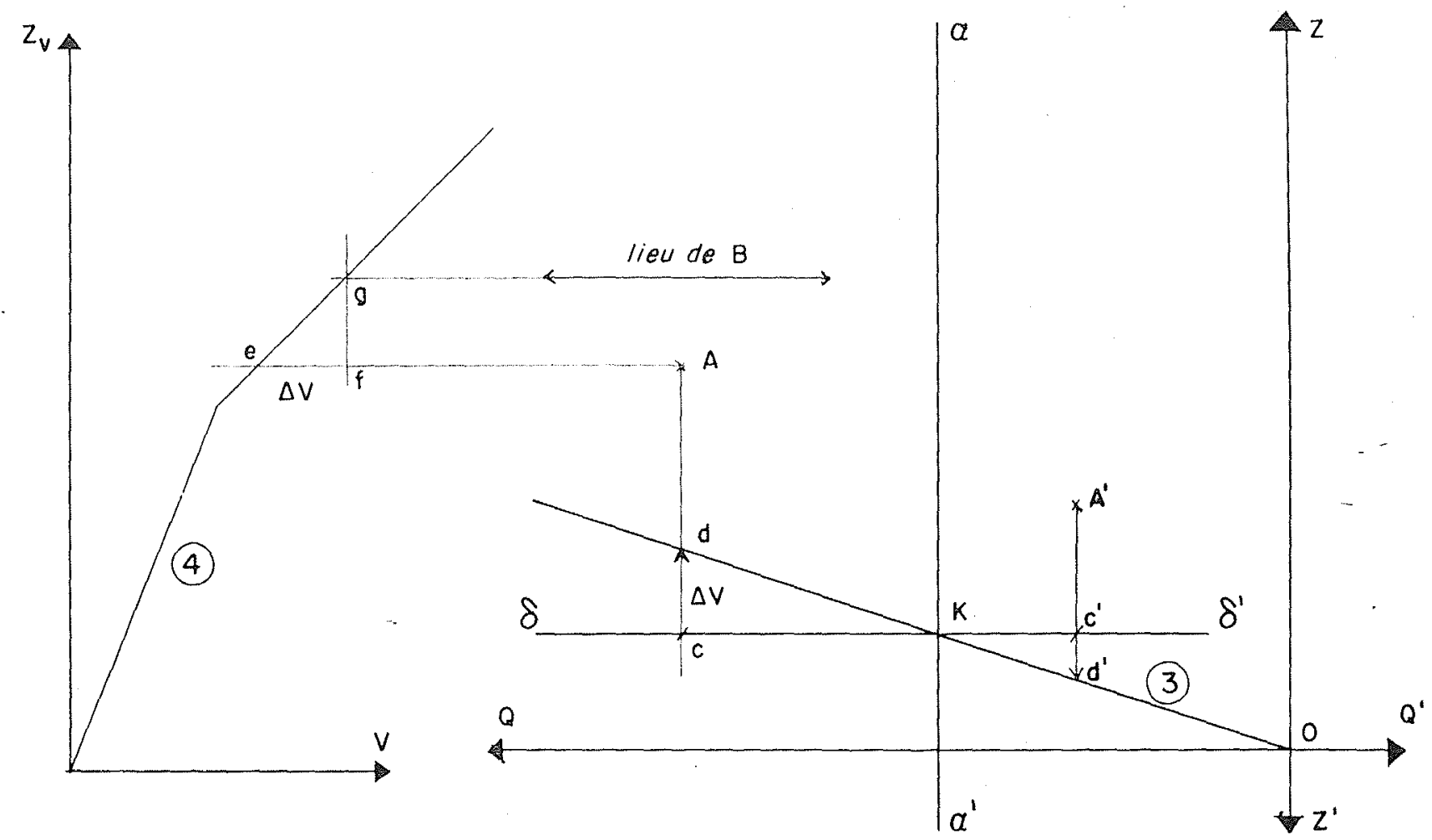

Fifi, 6 .

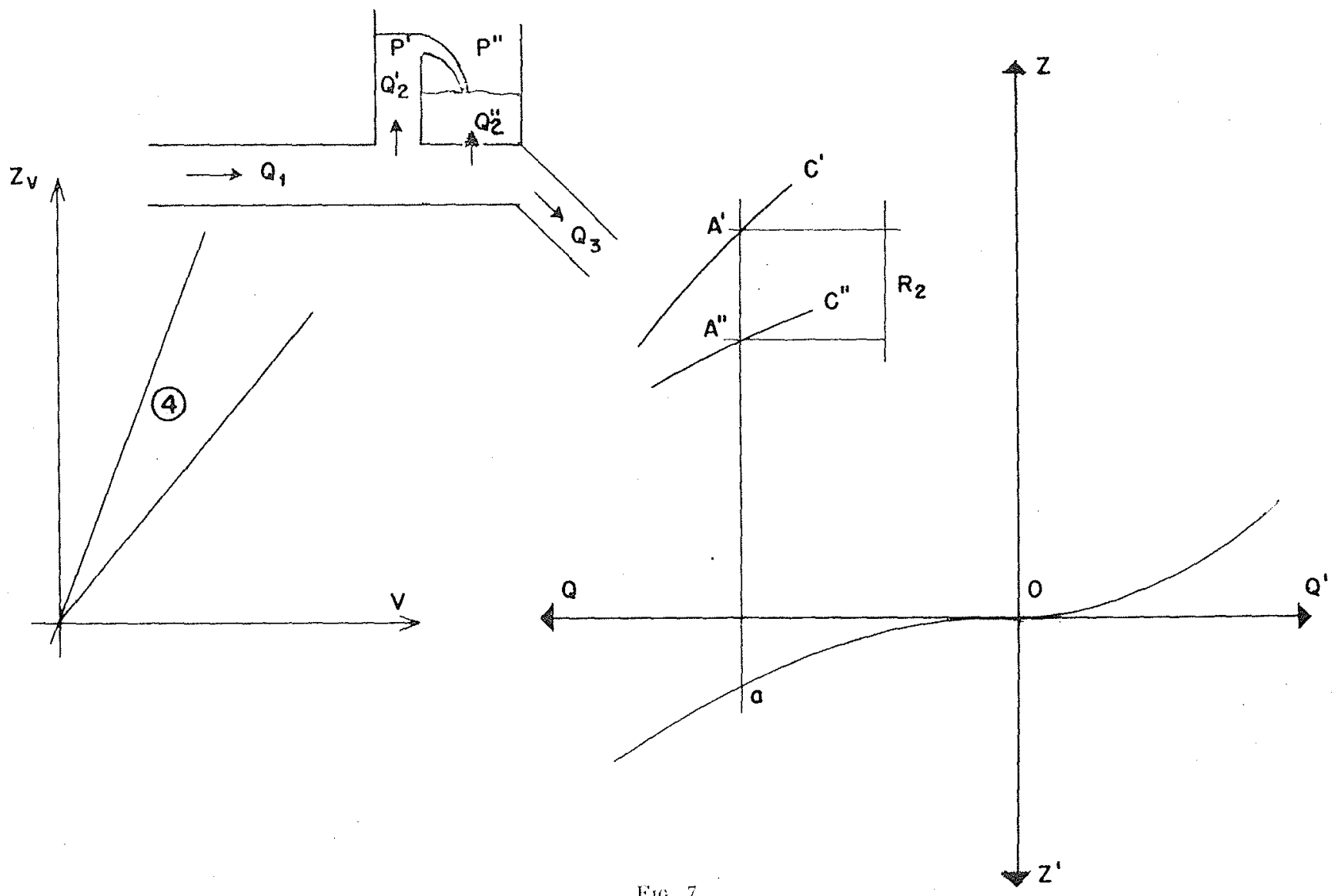

Fic. 7. 


\section{e) CAS PARTICUTER DES CHEMTNESS DIFEERTN- TIELLES.}

Les constructions sont un peu plus conpliquées par le fait que la cheminée se compose, en réalité, de deux puits $P^{\prime}$ et $P^{\prime \prime}$ dans lesquels passent, à un instant donné $t$, les débits $Q_{2}$ el $Q_{2}{ }^{\prime \prime}$ liés par $\mathrm{Q}_{2}{ }^{\prime}+\mathrm{Q}_{2}^{\prime \prime}=\mathrm{Q}_{2}$.

Le puits $P^{\prime \prime}$ possède à la base un étranglement créant une perte de charge $R_{2}$ pour le débit $Q_{2}{ }^{\prime \prime}$. La courbe (4) sera évidemment formée de deux lignes représentant le volume de chacun des puits en fonction de la cote (fig. 7).

La courbe représentative d'un fonctionnement de la cheminée sera formée également de deux lignes, $C^{\prime}$ et $C^{\prime \prime}$. Ainsi au point $A^{\prime}$ représentant l'état du puits $\mathrm{P}^{\prime}$ au temps $t$, correspond $\mathrm{A}^{\prime \prime}$ représentant $P^{\prime \prime}$ au même instant. $A^{\prime}$ et $A^{\prime \prime}$ ont même abscisse et la distance $A^{\prime} A^{\prime \prime}$ représente la perte de charge $R_{2}$ créée par l'etranglement.
Nous conseillons alors de disposer la courbe (1) représentant $R_{2}$ (n fonction de $Q_{2}$ ", comme il est indigue sur la figure 8 , ce qui permet d'obtenir facilement $Q_{2}^{\prime}$ et $Q_{. \prime \prime}^{\prime \prime}$, doù $\Delta V^{\prime}$ et $\Delta V^{\prime \prime}$. Le reste de la construction n'a rien de particulier.

\section{C) Méthode de deuxième approximation}

Yous renons le voir comment, connaissant le point $A$ a linstant $t$, on pouvait trouver le point $\mathrm{B}$ a l'instant $t+\Delta t$ en supposant que pendant le temps $\Delta t$ les quantités caractéristiques du point $A$ i $Q_{z}$ et $H_{a}$ ) restaient les mèmes et cigales à leurs raleurs au point $A$.

line deaxieme approximation consisterait is fairies deux opripations successives suivantes :

1. Construire le point $B$ à partir de $A$, comme on vient de l'indiquer, et en déduire le milieu M du segment AB;

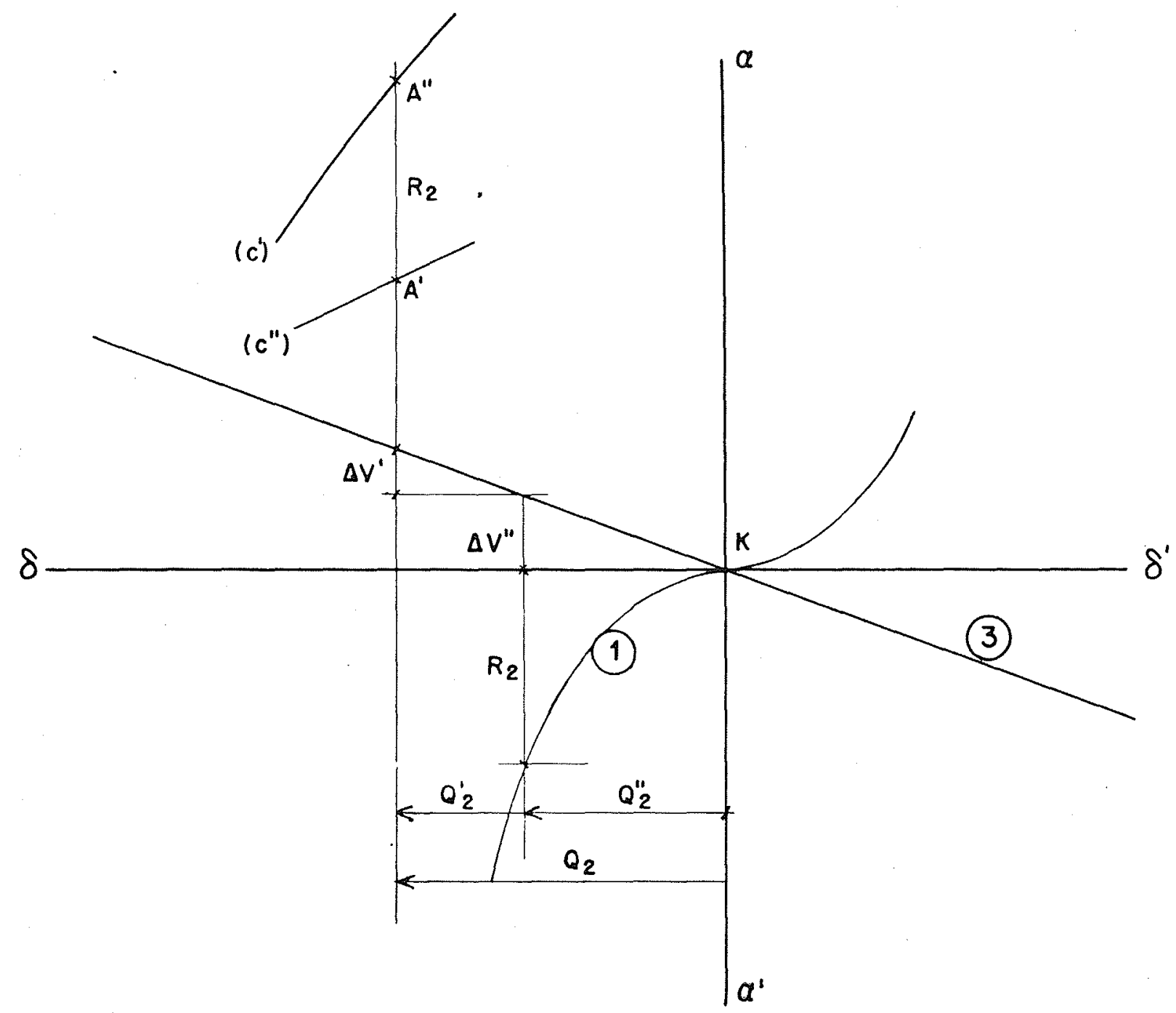

Fig. 8. 
2" Construire le point B' (très proche de B) à partir de A, en supposant que, pendant l'inter-

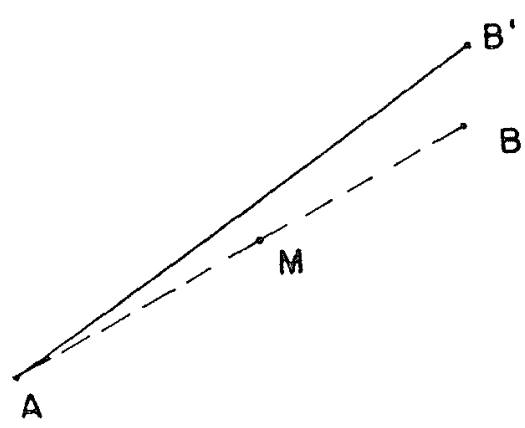

Fici. 9.

valle $\Delta t$, les conditions moyennes sont représentées par $\mathrm{Q}_{\text {z }}$ et $\mathrm{H}_{1}$ relatifs au point $\mathrm{M}$ (fig. 9).

Remarque. - L'erreur que l'on fait alor's est un infinment petit, d'un ordre de grandeur supérieur à celui de la première opération.

En réalité, on ne fait pas les deux opérations

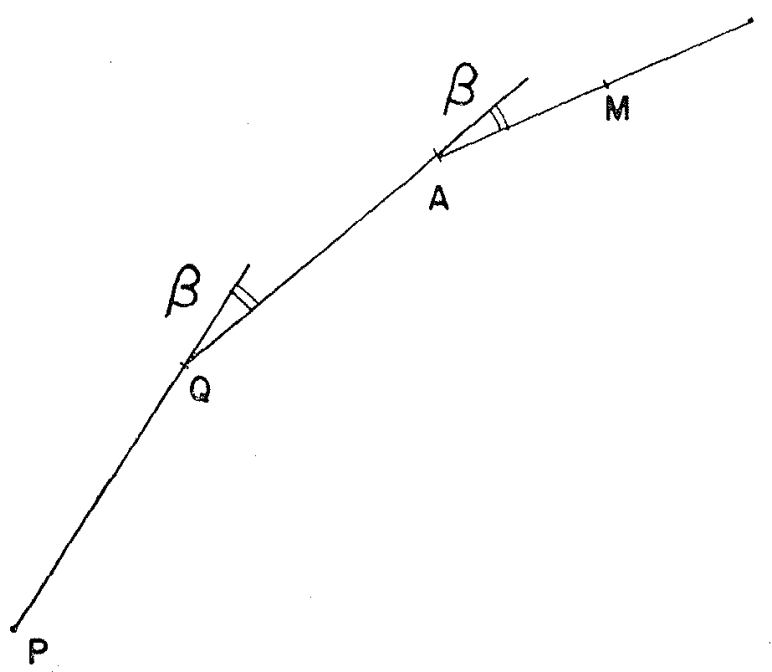

Fig. 10.

sucessives précédentes. Supposons déjà construits les segments PQ QA (fig. 10). L'expérience montre que la longueur de ces segments, ainsi que l'angle $\beta$ que deux segments font entre eux, varient très peu (et, de toutes façons, d'une maniere continue) d'un segment de droite à l'autre. Il est done facile, comnaissant P.Q.A., de suppuler l'emplacement du milieu $M$ de $A B$,

- soit au jugé, quand on en a l'habitude,

- soit en traçant le segment $\mathrm{AM}=\frac{\mathrm{QA}}{2}$ faisant un angle avec QA.

A notre avis dailleurs, la méthode qui consiste à estimer sans calcul la position du futur point M est meilleure, car les petites erreurs que fait l'opérateur à chaque supputation ne sont pas toutes systématiquement dans le mème sens. Il est donc très probable qu'elles se contrebalancent, ce qui a d'ailleurs écé vérifić par les épures que nous avons faites.

Au début d'un mouvement non permanent, il est évidemment impossible de supputer l'emplacement du milieu du segment AB; il suffit, dans ce cas, de faire les deux opérations successives indiquees plus haut (fig. 11).

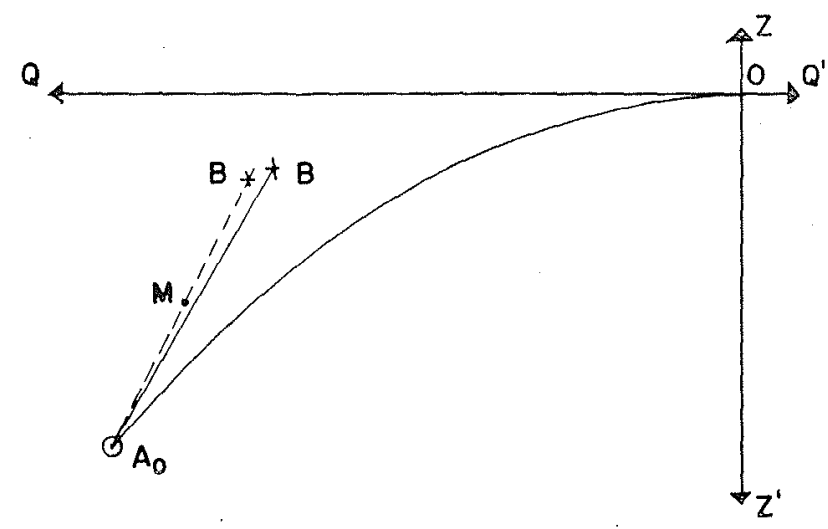

Fig. 11.

\section{D) Manœuvres non instantanées. Axe mobile}

L'axe mobile $x x^{\prime}$ permet d'étudier des manouvres de vannage non instantances.

Considérons, par exemple, une onverture totale en 30 secondes (fig. 12); si nous utilisons des $\Delta t$ de 10 secondes, il suffira de déplacer trois fois l'axe mobile $x x^{\prime}$. En effet, au temps 0 , $z x^{\prime}$ sera confondu avec laxe des coordonnées $Z^{\prime}\left(Q_{3}=0\right)$; au bout de 30 secondes, il sera ì gauche de cet axe, à la distance $Q$.: de $O^{\prime}\left(Q_{3}\right.$ représentant le débit passant dans les turbines à la fin de la manœuve d'ouverture). Pratiquement, pendant les dix premieres secondes, l'axe $\alpha x^{\prime}$ se déplaçant de $O^{\prime}$ au point a (figure 12), nous ferons les

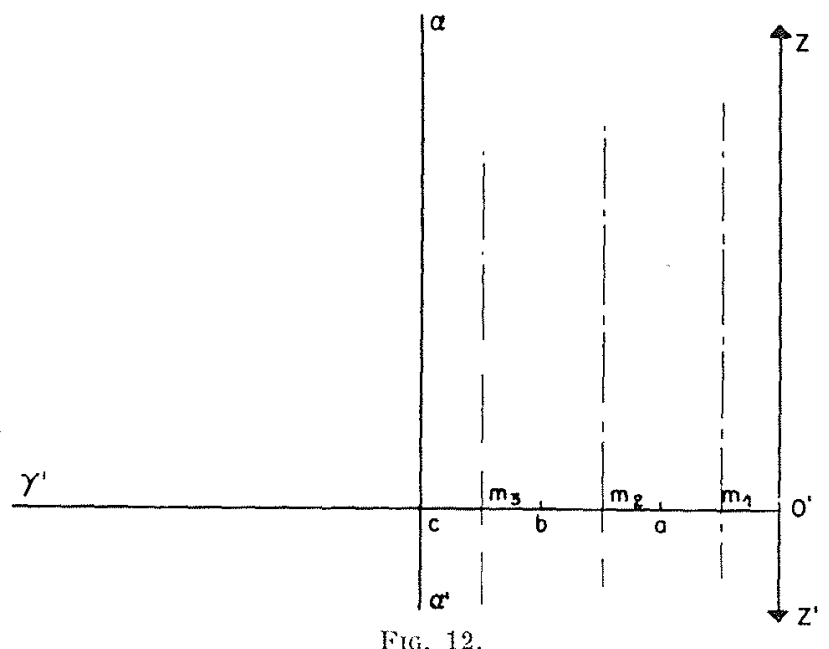


constructions habituelles en supposant qu'il reste fixe et coupe $O^{\prime} \gamma^{\prime}$ au point $m_{1}$ milieu de $O^{\prime} a$ - pendant les dix secondes suivantes, il sera également supposé immobile et coupera $\mathrm{O}^{\prime} \gamma^{\prime}$ en $m_{2}$ milieu de $a b$, elc...

\section{E) Choix de l'intervalle $\lrcorner t$}

On prend, en général, $\Delta t=5,10$ ou 20 secondes; cela dépend du degré de précision cherché et des caractéristiques du système galerie-cheminée d'équilibre.

Si on prend un $\Delta t$ trop petit, les opérations deviennent longues et fastidieuses. Si on prend un $\Delta t$ trop grand, la précision du graphique risque de s'en ressentir. Ce n'est qu'après avoir fait quelques épures que l'opérateur pourra apprécier à l'avance le $\Delta t$ le plus adéquat.

Il est d'ailleurs tres facile de changer de $\Delta t$ et il esí mème souvent conseillé de le faire au cours d'une épure; en effet, à mesure que le mouvement s'amortit, les segments représentatifs des temps $\Delta t$ diminuent et il est commode de prendre un $\Delta t$ deux ou quatre fois plus grand. Pour cela, il suffit simplement de multiplier par 2 ou 4 la pente des droites des graphiçues (2) et (3).

Dans certaines opérations, il est mème commode de prendre momentanément des $\Delta l$ tout à fait quelconques, comme nous le verrons plus loin dans les épures concernant les cheminées déversantes ou différentielles.

\section{F) Construction de la courbe $Z_{v}$ en fonction du temps}

Cette courbe se déduit immédiatement des épures en $Z_{r}$ et $Q_{1}$ dont nous venons de parter, puisque nous connaissons, en chaque point de la ligne représentative du mouvement, le temps et le niveau de l'eau dans la cheminée d'équilibre.

\section{IV. - EXEMPLES D'ÉPURES}

\section{A) Cheminée d'équilibre}

à chambres d'expansion (épure $n^{\circ}$ 1)

Exemple. - Cheminée d'équilibre de la chute de Passy - Epure de fermeture totale instantanée:

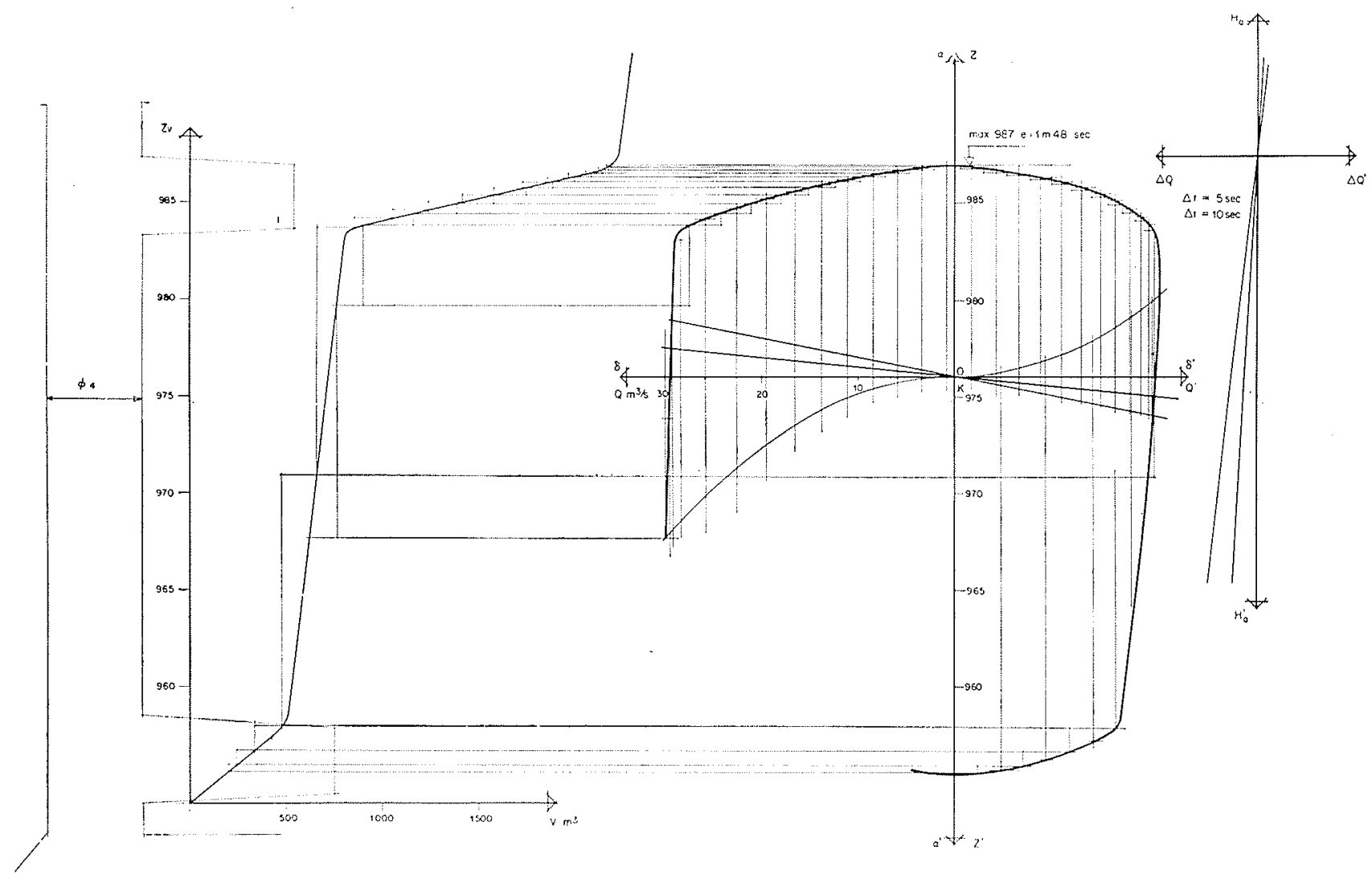




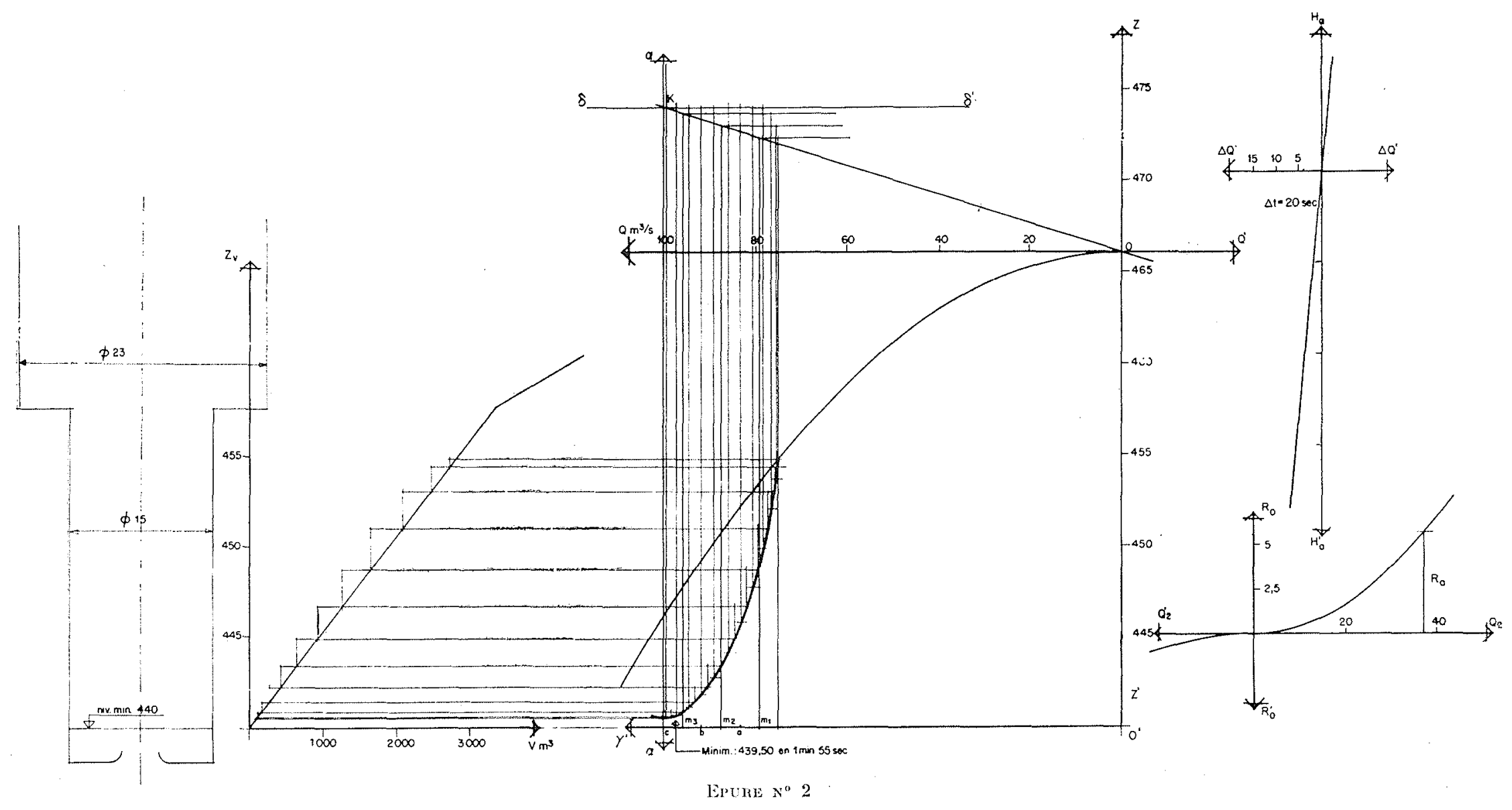

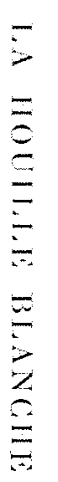

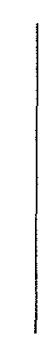




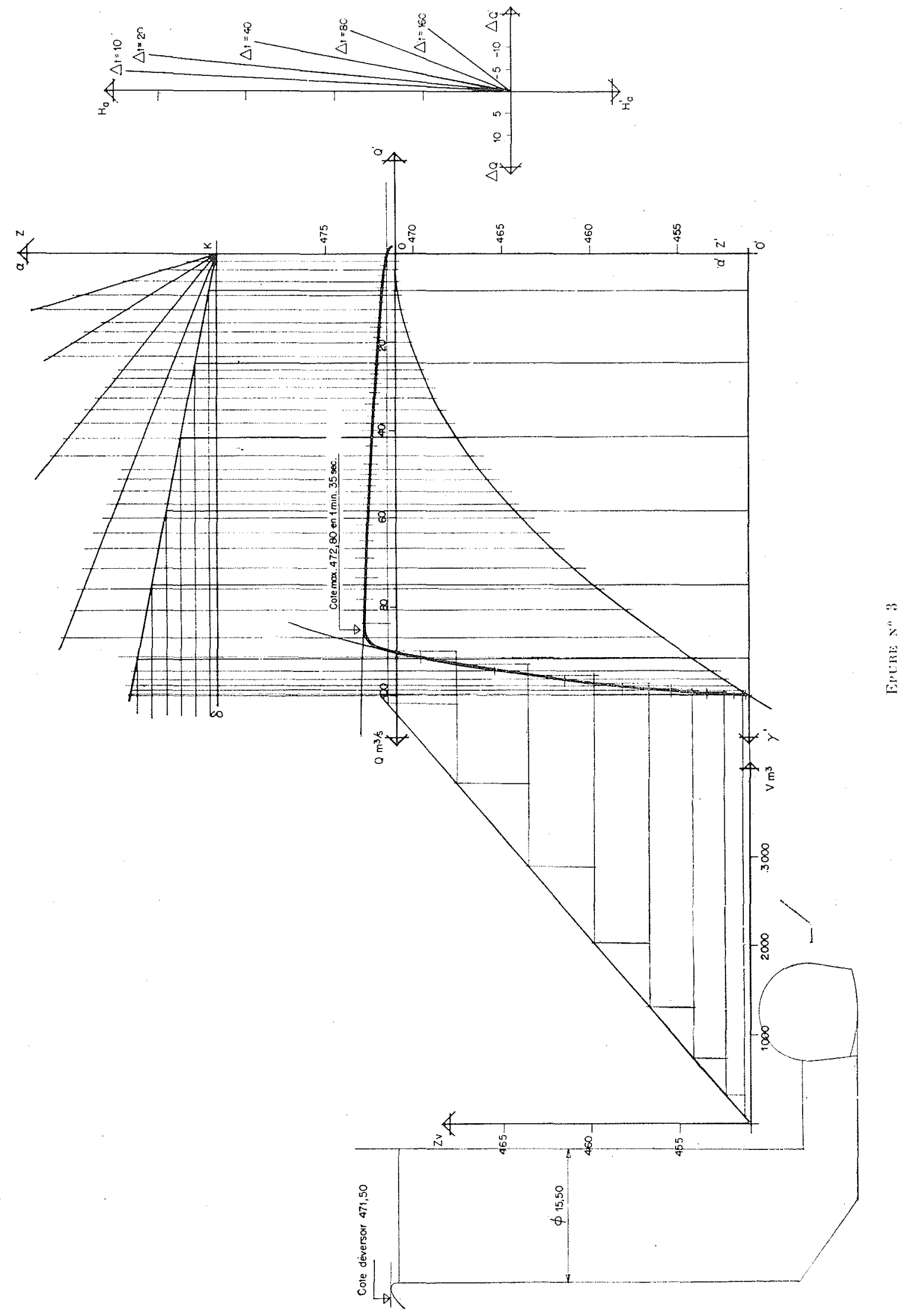


Caractéristiques

$$
\begin{aligned}
\mathrm{Q}_{1} & =30 \mathrm{~m}:: \mathrm{s} . \\
\mathrm{L}_{1} & =5.885 \mathrm{~m} . \\
f & =13,70 \mathrm{~m}^{2} . \\
\mathrm{Z}_{0} & =976 . \\
\mathrm{R}_{1} & =8,30 \mathrm{~m} \text { pour } Q_{1}=30 \mathrm{~m}: 3 / \mathrm{s}
\end{aligned}
$$

$\lambda t$ ulilisés $=5$ et 10 secondes.

\section{B) Cheminée d'équilibre à étranglement} (épure $n^{\prime \prime} 2$ )

\section{EXemple. - Cheminée d'équilibre} de la chute de Randens

- Epure d'ouverture linéaire de 75 à $100 \%$ (temps d'ouverture : 1 minute).

\section{Caractéristiques}

$$
\begin{aligned}
\mathrm{Q}_{1} & =100 \mathrm{~m}: \mathrm{s} . \\
\mathrm{L} & =16.144 \mathrm{~m} . \\
f & =31,10 \mathrm{~m}^{2} . \\
\mathrm{R}_{1} & =20 \mathrm{~m}, \text { pour } \mathrm{Q}=100 \mathrm{~m} / \mathrm{s} . \\
\mathrm{R}_{2} & =40 \mathrm{~m} \text { (pour } \mathrm{Q}=100 \mathrm{~m} / \mathrm{s}) . \\
Z_{0} & =466 .
\end{aligned}
$$

$\Delta t$ utilisé $=20$ secondes.

\section{C) Cheminée d'équilibre déversante}

(épure $n^{\circ} 3$ )

Exemple. - Adapté à la chute de Randens

-.. Epure de fermeture linéaire totale en 1 minute.
Caracteristiques

$$
\begin{aligned}
\mathrm{Q}_{1} & =100 \mathrm{~m}: \mathrm{s} . \\
\mathrm{L}_{1} & =16.144 \mathrm{~m} . \\
f & =31,10 \mathrm{~m} \% \\
\mathrm{Z}_{0} & =471 . \\
\mathrm{R}_{1} & =20 \mathrm{~m} \text { pour } \mathrm{Q}_{1}=100 \mathrm{~m} \% / \mathrm{s}
\end{aligned}
$$

$\Delta t$ utilisés $=10,20,40,80,160$ secondes.

\section{Remarques :}

1. La partie supérieure de la courbe n'est pas autre chose que la courbe caractéristique (5) du déversoir de la cheminée; elle est de la forme $\mathrm{Q}=\mathrm{K} h^{: / 2 / 2}, h$ étant la hauteur de leau au-dessus du déversoir.

2. Le volume d'eau déversé se mesure aisément en additionnant tous les segments découpés entre les droites (3) et laxe des Q.

Dans le cas de l'épure, il est de $V=45.000 \mathrm{~m}^{*}$.

\section{D) Cheminée d'équilibre différentielle}

(épure $n^{\circ} 4$ )

Exemple. - Adapté à la chute de Randens

- Epure de fermeture totale instantanće.

Caractéristiques

$$
\begin{aligned}
\mathrm{Q}_{1} & =100 \mathrm{ml}^{3} / \mathrm{s} . \\
\mathrm{L} & =16.144 \mathrm{~m} . \\
f & =31,10 \mathrm{~m}^{2} . \\
\mathrm{R}_{1} & =15 \mathrm{~m} \text { pour } \mathrm{Q}=100 \mathrm{~m}^{3} / \mathrm{s} \\
\mathrm{R}_{2} & =90 \mathrm{~m} \text { pour } \mathrm{Q}=100 \mathrm{~m}^{3} / \mathrm{s} \\
\mathrm{Z}_{0} & =471 .
\end{aligned}
$$

$\Delta t$ utilisés $=20$ el 4 secondes.

\title{
EXTENSION A UNE CHEMINEE D'EQUILIBRE BRANCHEE SUR UNE GALERIE EN $\mathbf{Y}$
}

\author{
(N.S. DIFFÉRINTSS)
}

Considérons une cheminée d'équilibre branchce sur une galerie en $\mathrm{Y}$ reliée à deux prises d'eau à des niveaux statiques différents fixes, représentéc sur la figure 13.

Désignons par :

$Q_{1}$, le débit de la galerie principale,

$q^{\prime}$ et $q^{\prime \prime}$, le débit des galeries secondaires,

$f^{\prime} f^{\prime \prime}$ et $f$, les sections des galeries,

$z_{0}^{\prime}$ et $z_{0}^{\prime \prime}$ les niveaux statiques aux prises d'eau,

$r^{\prime}$ et $r^{\prime \prime}$ les pertes de charge des galeries secondaires,

$R_{1}$, la perte de charge de la galerie principale.

Les autres notations seront celles de la première partie. 

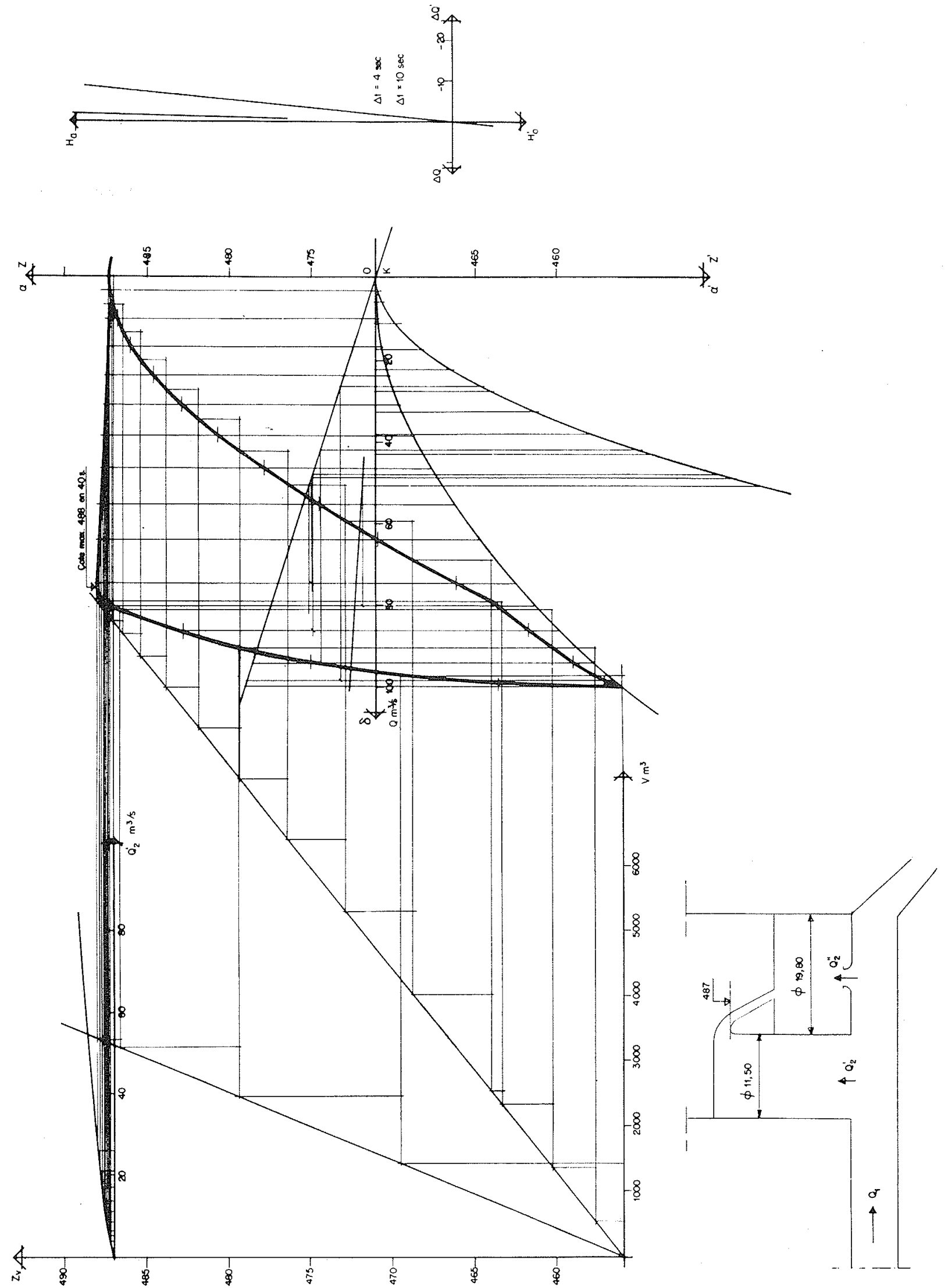

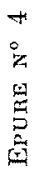




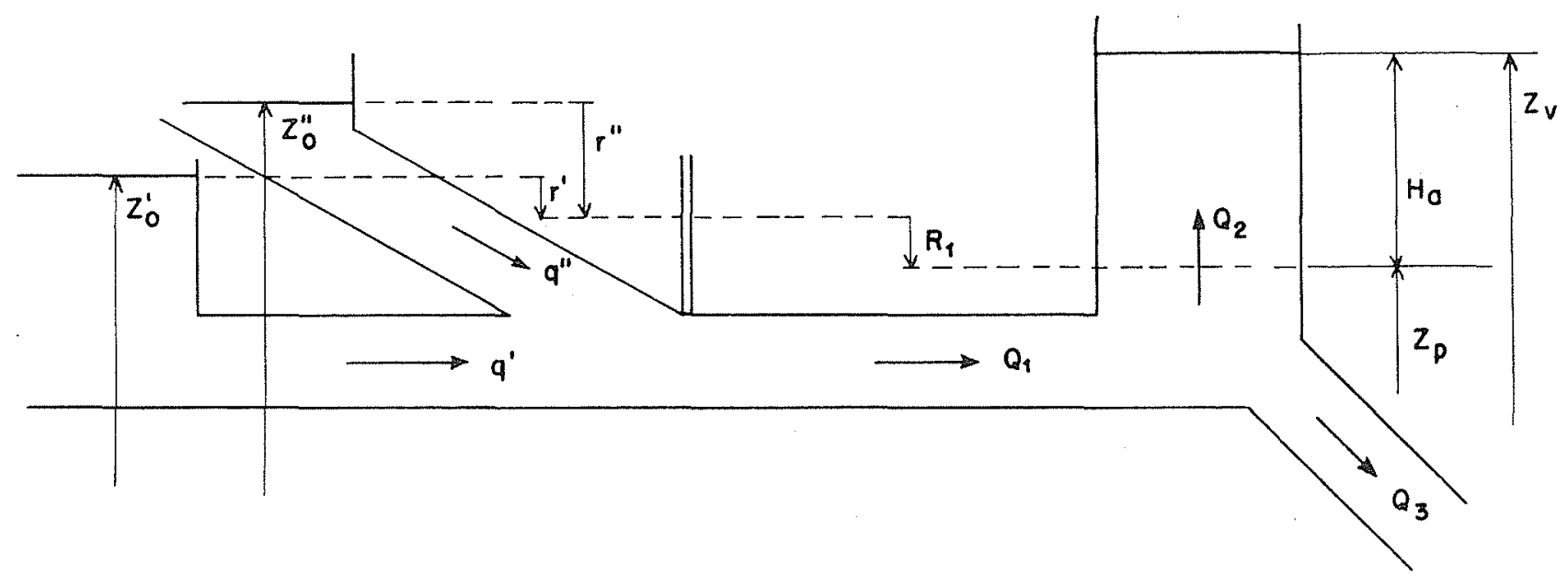

Fig, 13.

\section{I. - PRINCIPE}

Nous imaginerons qu'il existe, au confluent des trois galeries appele "point triple», un tube piézométrique de section très petite, qui se bornerait à enregistrer les pressions. Supposons commus, à un instant, les débits dans chacune des galeries, ainsi que le niveau à la chemince d'équilibre et la pression au point triple.

Examinons comment ce systeme de trois galeries peut être décomposé en éléments simples qui nous raminent an cas du systeme galerie unicfue-cheminée d'épuilibre.

\section{A) Galeries secondaires}

Les variations de débit de ces galeries sont les mêmes que si clles étaient alimentées par les prises d'eau, le tube piézométrique jount à leur égard le ròle de cheminée d'équilibre. Ceci posé, les hauteurs accélératrices se calculent comme au chapitre II.

\section{B) Galerie principale}

Le niveau dans le tube piézométrique joue, à l'instant $t$, le rôle de niveau à la prise d'eau dans un système classique galerie-cheminée d'équilibre, d'où le calcul de $\mathrm{H}_{a}$ relatif à ce troncon.

Ces diverses hauteurs accélćratrices, $h_{t}{ }^{\prime}, h_{t}{ }^{\prime \prime}, \mathrm{H}_{t}$ entraineront, durant l'intervalle du temps $\Delta t$, des variations de débit données par :

$$
\begin{aligned}
& \Delta q^{\prime}=\frac{g f^{\prime}}{l^{\prime}} h_{a}^{\prime} \Delta t=k^{\prime} h_{a^{\prime}} \Delta t \\
& \Delta q^{\prime \prime}=\frac{g f^{\prime \prime}}{l^{\prime \prime}} \cdot h_{{ }^{\prime \prime}}{ }_{a} \Delta t=k^{\prime \prime} h_{a}{ }^{\prime \prime} \Delta t
\end{aligned}
$$

$$
\lrcorner Q_{1}=\frac{g f}{\mathrm{~L}} \quad \mathrm{H}_{a} \Delta t=\mathrm{KH}_{u} \Delta t
$$

Les débits ef leurs variations devront satisfaire en outre l'équation de continuité.

$$
\begin{aligned}
\mathrm{Q}_{1} & =q^{\prime}+q^{\prime \prime} \\
\Delta \mathrm{Q}_{1} & =\Delta q^{\prime}+\Delta q^{\prime \prime}
\end{aligned}
$$

Nous allons exposer une méthode graphique, qui permettra, à partir de ces égalités, de déterminer la montée de l'eat dans la chemince d'équilibre en cas de fermeture ou d'ouverture des turbines. Nous n'examinerons que les manouvres brusques, mais les manouvres simples (ouvertures ou fermetures non instantanées) pourront être ramenées à ce cas particulier, en utilisant l'axe mobile indiqué précédemment.

Lapplication de cette methode peut comporter deux stades dapproximation que nous examinerons successivement :

1. Elape de premiere approximation laissant subsister l'erreur systématique existant dans les méthodes Schurmin-ied Felice pour les chminées simples. Les variables, au cours d'un instant $\Delta t$, sont supposées conserver les valeurs qu'elles avaient au début de cet instant;

2. Nous verrons qu'il est ensuite facile de passer à la seconde approximation en éliminant cette erreur systématicue.

\section{II. - CONSTRUCTION D'UNE ÉPURE}

\section{A) Disposition de l'épure}

Le fonctionnement du systeme sera examiné dans un graphique représentant en ordonnce le 
niveau dans la cheminée, et, en abseisse, les débits dans les galeries secondaires et principales. La cote de l'eau dans la cheminée sera représenlée en fonction du débit total $Q_{1}$. Nous représenterons, en outre, les niveaux piézométriques en régime permanent de l'extrémité aval des deux galeries secondaires à leurs cotes respectives, en portant, a partir des cotes statiques, les pertes de charge en fonction du débit, représentées par des paraboles. Nous nous limiterons, pour la clarté de l'exposé, au cas où la cheminée d'équilibre ne comporte pas d'étranglement, mais le principe de l'ćtude est le même quel que soit le type de cheminée.

Les pertes de charge dans la galerie principale seront représentées, en fonction du débit, en dessous des paraboles précédentes, à une distance quelconque des niveaux statiques des prises d'eau (fig. 14).

En régime permanent, les points de fonctionnement des galeries secondaires seront sur les paraboles correspondantes, et sur une même parallèle à l'axe des abscisses. Il en sera autrement en régime transitoire.

Fixons-nous à l'instant $t$ :

- les débits $q^{\prime}, q^{\prime \prime}, \mathrm{Q}_{1}$,

- le niveau dans la cheminée d'équilibre, qui déterminent un point représentatif du mou. roment de coordonnées $Q_{1}$ et $Z$ à l'instant $t$.

Nous allons déterminer :

- la pression au point triple à l'instant $t$,

- les débits $q^{\prime}, q^{\prime \prime}, Q_{1}$ a l'instant $t+\Delta t$,

-.. le niveau dans la cheminée d'écuilibre à linstant $t+\Delta t$,

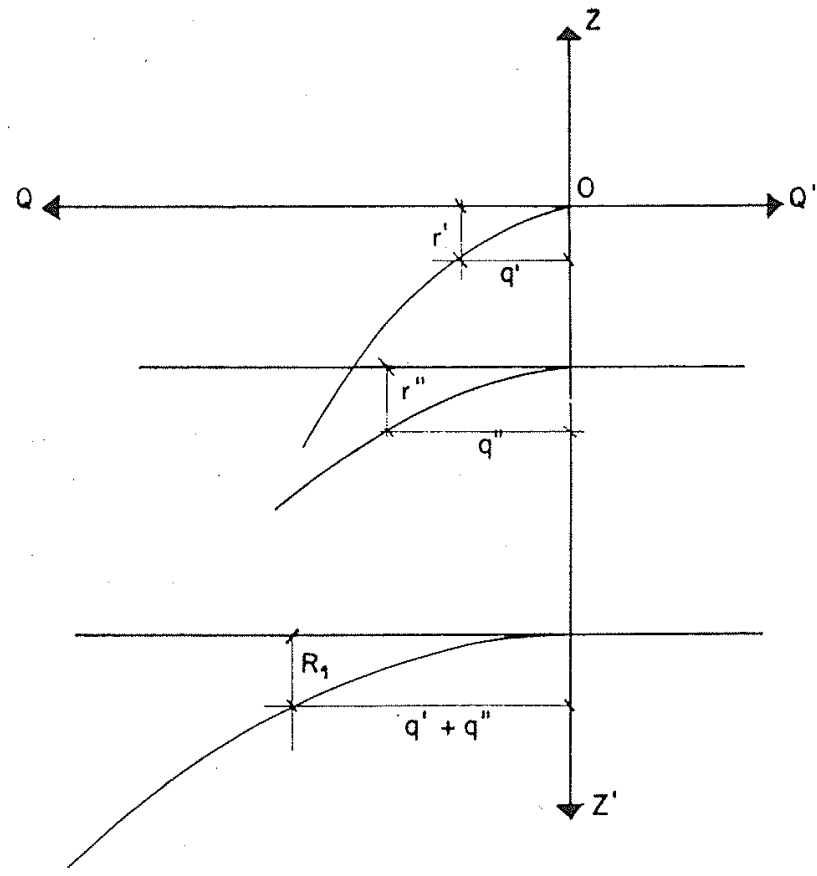

Fit. 14 .

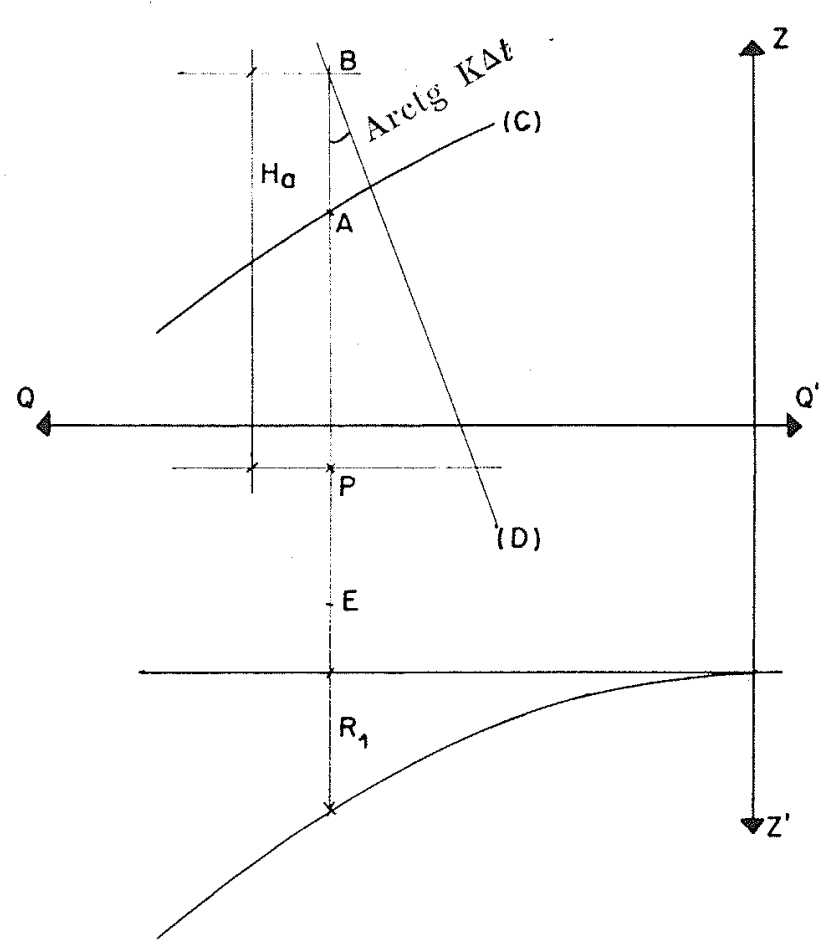

Fili. 15.

qui déterminent le point de fonctionnement à l'instant $t+\Delta t$.

Soit le point representatif $\mathrm{A}$, a l'instant $t$.

Supposons connue, pour la commodité de l'exposé, la pression au point triple, et représentons-la par le point $P$ dans te graphicue.

\section{B) Détermination de $\Delta Q$}

1" Galerie principale. -... Conformément au partage en éléments simples, la hauteur accélératrice de cette galerie s'obtiendra de la façon suivante :

On détermine le niveau de régime permanent en retranchant ou en ajoutant (suivant le sens de l'écoulement dans la galerie) les pertes de charge $R_{1}$, de la pression au point triple, ce qui donne le nouveau point E. La distance verticale EA représente la hauteur accélératrice $\mathrm{H}_{0}$ de la galerie principale; il revient au même et il est préférable pour la commodité du graphique de calculer $\mathrm{H}_{t}$, en ajoutant au niveau de la cheminée les pertes de charge dans la galerie principale, ce qui donne le point B. La distance BP représente alors la hateur accélératrice $\mathrm{H}_{a}$ (figure 15 ).

Portons alors, a partir du point B, une droite de coefficient angulaire $\mathrm{K} \Delta t$, par rapport à la verticale. La distance horizonlale détermince enIre cette droite el la verticale de $B$ sur une horizontale passant par $P$ représente la variation 
de débit dans la galerie principale durant l'inlervalle de temps $\Delta t$.

2" Galeries saconidaris. - Les hauteurs accélératrices respectives se déduisent des niveaux de régime permanent, comme dans un système classique. Elles sont représentée par $h_{a}{ }^{\prime}$ et $h_{q}{ }^{\prime \prime}$ dans la figure 16. Menons les droites $d^{\prime}$ et $d^{\prime \prime}$ faisant, avec la verticale de $P$, des angles dont les tangentes seront respectivement égales à $k^{\prime} \Delta t$ et $k^{\prime \prime} \Delta t$. On aura alors $\Delta q^{\prime}$ et $\Delta q^{\prime \prime}$, comme l'indique la figure 16.

\section{C) Détermination de la pression au point triple}

En réalité, la position du point $P$ est inconnue, mais l'équation de continuité va nous permettre de la déterminer, et, par là, de connaître $\Delta Q_{1}, \Delta q^{\prime}$ et $\Delta q^{\prime \prime}$.

Soit $A$ le point représentatif de l'état de la cheminée d'équilibre à l'instant $t$.

Portons, à partir de $\mathrm{A}$, les pertes de charge $\mathrm{R}_{1}$, d'oì le point $B$, et menons par $B$ la droite $D$ de coefficient angulaire $\mathrm{K} \Delta t$ par rapport à la verticale.

Menons, à partir de $i^{\prime}$ et de $i^{\prime \prime}$ situés à l'intersection de la verticale du point $A$ avec le nitveau de régime permanent des galeries secondaires, les droites $d^{\prime}$ et $d^{\prime \prime}$, de coefficients angulaires $k^{\prime} \Delta t$ et $k^{\prime \prime} \Delta t$ du même côté par rapport à $\mathrm{BB}^{\prime}$ que la droite D. Additionnons les abscisses de $\left(d^{\prime}\right)$ et de $\left(d^{\prime \prime}\right)$ comme indiqué sur la figure 17. L'intersection de (D) avec $\left(d^{\prime}+d^{\prime \prime}\right)$ se produit à une cote représentant précisément la pression du point triple puisqu'on a, par consiruction :

$$
\Delta Q_{1}=\Delta q^{\prime}+\Delta q^{\prime \prime}
$$

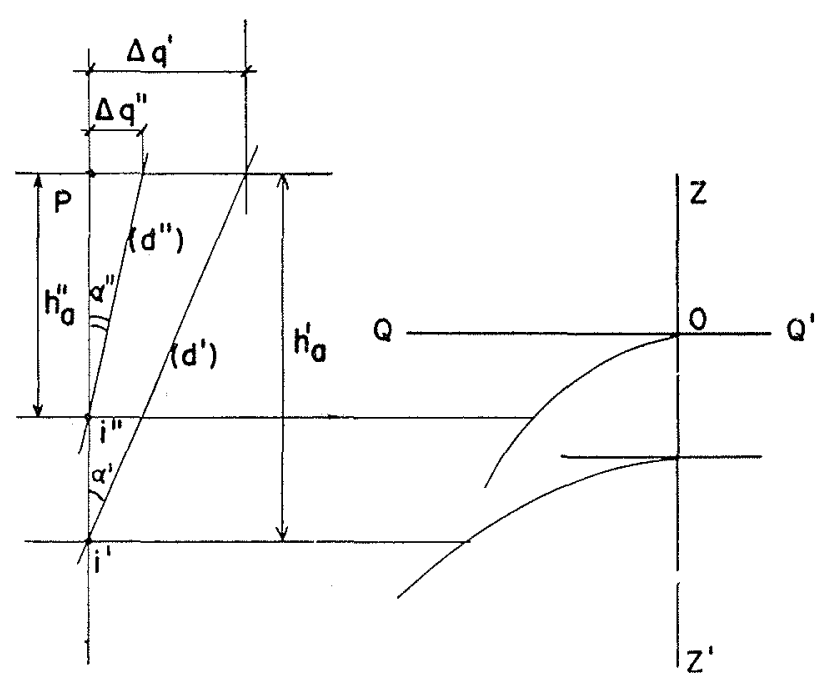

Fic. 16 ,

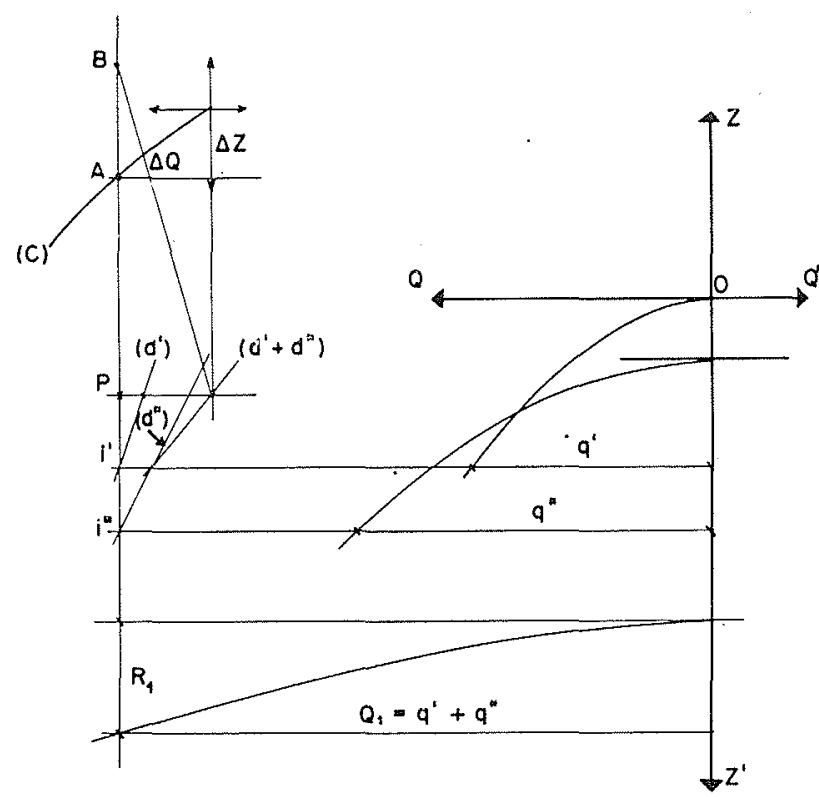

Fig. 17.

Pratiquement, on mène d'abord la droite issue du point $i$ le plus bas ( $i^{\prime \prime}$ dans le cas de la figure), puis, à partir de son intersection avec l'horizontale de $i^{\prime}$, une droite de coefficient angulaire $\left(k^{\prime}+k^{\prime \prime}\right) \Delta t$.

On retranche ensuite les variations de débit $\Delta Q_{1}, \Delta q^{\prime}$ et $\Delta q^{\prime \prime}$ des débits respectifs a l'instant $t$, ce qui donne les nouvelles valeurs de ces débits à l'instant $t+\Delta t$. Pratiquement, on a intérèt, pour la précision du graphique, à reporter les deux variations les plus importantes, soit, par exemple, $\Delta Q_{1}$ et $\Delta q^{\prime \prime}$, et à en déduire le débit $q^{\prime}$ par différence.

\section{D) Déćermination de $\Delta Z$}

La méthode est la même que pour une cheminée d'équilibre branchée sur une galerie simple. On a encore :

$$
\Delta Z=\frac{Q_{2}}{\mathrm{~F}} \Delta t
$$

avec les notations des autres chapitres. La montée se détermine donc à l'aide des mêmes constructions graphiques que précédemment (courbes (3) et (4) des exposés précédents).

Le report de $\Delta Q_{1}$ et de $\Delta Z$ permet de déterminer le point de fonctionnement à l'instant $t+\Delta t$.

\section{E) Seconde approximation}

Notis venons de voir comment, connaissant le point A à linstant $t$, on pouvait trouver le point $A^{\prime}$ à l'instant $t+\Delta t$ en supposant que, pendant l'intervalle de temps $\Delta t$, les quantités 


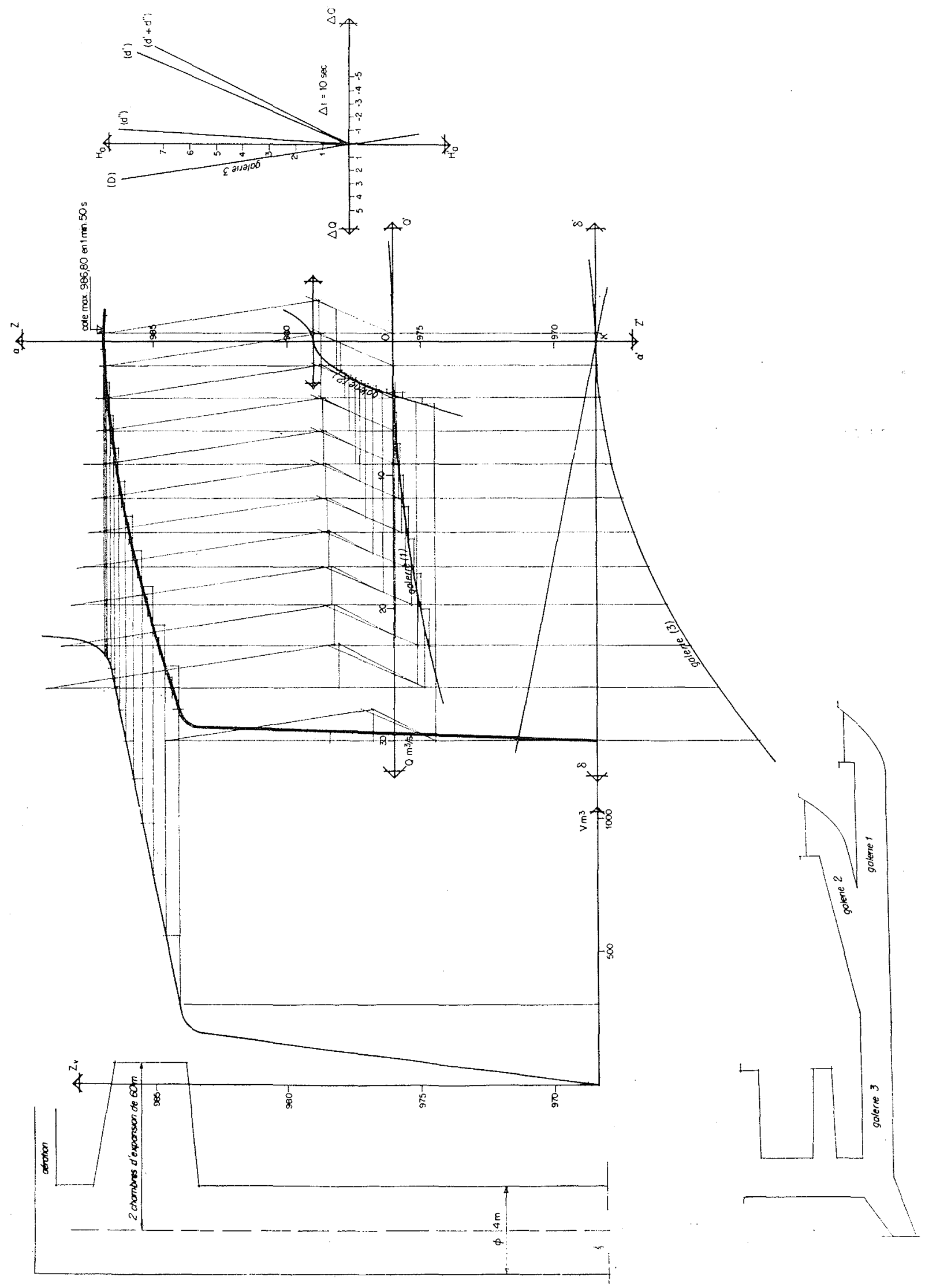

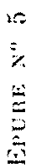


caracteristiques $\mathrm{Q}_{1}$ et $\mathrm{H}_{t}$ restaient les mèmes of égales à leurs valeurs au point $A$.

La seconde approximation consiste à opcirer par réitération, en construisant, à partir de $A$, le nouveau point $A^{\prime}$, et 'n le précisant en prenant les quantités caractéristiques du milieu $\mathrm{M}$ de $\mathrm{AA}^{\prime}$.

L'expérience montre d'ailleurs, sauf cas particulier où la hauteur accélératrice varie beaucoup au cours d'un intervalle $\Delta t$, cu'il est possible d'estimer directement la position des milicux des segments intervenant dans la construction, ce qui permet de déterminer $\mathrm{A}(t+\Delta t)$ par une seule operation.
Lépure que nous donnons on annexe (5) a dé réalisée suivant cette méthode. Elle concerne la cheminée à expansion de la chule de PAssy, compte tenu de l'adduction complémentaire de la Drosaz. Les caractéristiques des trois galeries sont les suivantes:

$$
\begin{aligned}
\mathrm{Q}_{\mathrm{t}} & =30 \mathrm{~m} \cdot / \mathrm{s} \\
\gamma^{\prime} & =25 \mathrm{~m}^{3} / \mathrm{s} \\
\gamma^{\prime \prime} & =5 \mathrm{~m}^{\circ} / \mathrm{s} \\
f^{\prime} & =13,7 \mathrm{~m}^{2} \\
f^{\prime \prime} & =5 \mathrm{~m}^{2}
\end{aligned}
$$

\title{
CAS PARTICULIER
}

\section{CHEMINEE D'EQUILIBRE، BRANCHEE SUR UNE GALERIE EN V}

\author{
(N.S. DIFFERENTS)
}

Diflérents procédés de calcul concernant les cheminées branchées sur des galeries de ce type avaient été proposés à propos de la cheminée. d'équilibre de l'usine de Brogr.

1 "M. V. Goutrin a admis que, en régime permanent et en régime transitoire, la différence des pertes de charge des deux galeries reste constante et égale à la dénivellation existant entre les deux prises d'eau. On peut alors remplacer, dans les calculs, les deux galeries par une galerie écpuivalente qui permet de rentrer dans le cadre des calculs classiques de MM. Calame et Gaden. Cependant, cette méthode présente l'inconvénient de reposer sur une hypothèse approximative, comme nous le verrons plus loin.

$2^{\prime}$ M. L. Escande a eflectué le calcul en appliquant une méthode d'intégration par différences finies, qui présente certains inconvénients : elle demande beaucoup de temps, ne permet de traiter que des cas simples, non seulement pour les manœuvres envisagées (fermetures ou ouvertures complètes instantanées), mais aussi pour le type de cheminée (le cas des cheminées à section multiple doit lui-même ètre ramené par un artifice de pondération au cas des cheminées a une seule section). Enfin, son approximation equivaut à celle de la méthode graphique « de première approximation ».

Dans le but de trouver une méthode qui ne présente pas les inconvénients précités, nous avons essayć d'adapter la construction graphique précédente à ce cas particulier.

La décomposition en éléments simples, relative aux galeries en $Y$, reste valable, mais on n'a plus besoin de faire appel à l'équation de continuité pour déterminer les variations de débit.

La disposition de l'épure est analogue à celle des galeries en $\mathrm{Y}$ : représentation des niveaux statiques et des pertes de charge dans les galeries; étude du mouvement dans un système de coordonnées, niveau dans la cheminée d'équilibre, débit total de deux galeries. Le tube piézométrique précédent est ici remplacé par la cheminée elle-même, et la recherche de la hauteur: accélératrice résulte des mêmes opérations que dans les cheminées branchées sur galerie simple.

Nous donnons ci-joint (épure n" 6) une épure concernant une fermeture linéaire en 40 secondes d'une installation fictive analogue à celle de Broge dont les caractéristiques sont les suivantes :

Fermeture linéaire en $40 \mathrm{~s} . \Delta t$ utilise $=5 \mathrm{~s}$.

$$
\begin{aligned}
q^{\prime} & =10 \mathrm{~m}^{3} / \mathrm{sec} . \\
q^{\prime \prime} & =15 \mathrm{~m}^{\circ} / \mathrm{sec} . \\
f^{\prime} & =3,6 \mathrm{~m}^{2} \\
f^{\prime \prime} & =6 \mathrm{~m}^{2} \\
z_{0}{ }^{\prime}-z_{0}^{\prime \prime} & =5 \mathrm{~m} \\
r^{\prime} & =10 \mathrm{~m} \text { pour } q^{\prime}=10 \mathrm{~m}: / \mathrm{sec} . \\
r^{\prime \prime} & =5 \mathrm{~m} \text { pour } q^{\prime \prime}=15 \mathrm{~m}^{3} / \mathrm{sec} .
\end{aligned}
$$

Nous avons tracé la courbe donnant la différence des pertes de charge entre les deux galeries, en fonction du temps, ce qui nous a montré qu'elle ne restait pas constante. 

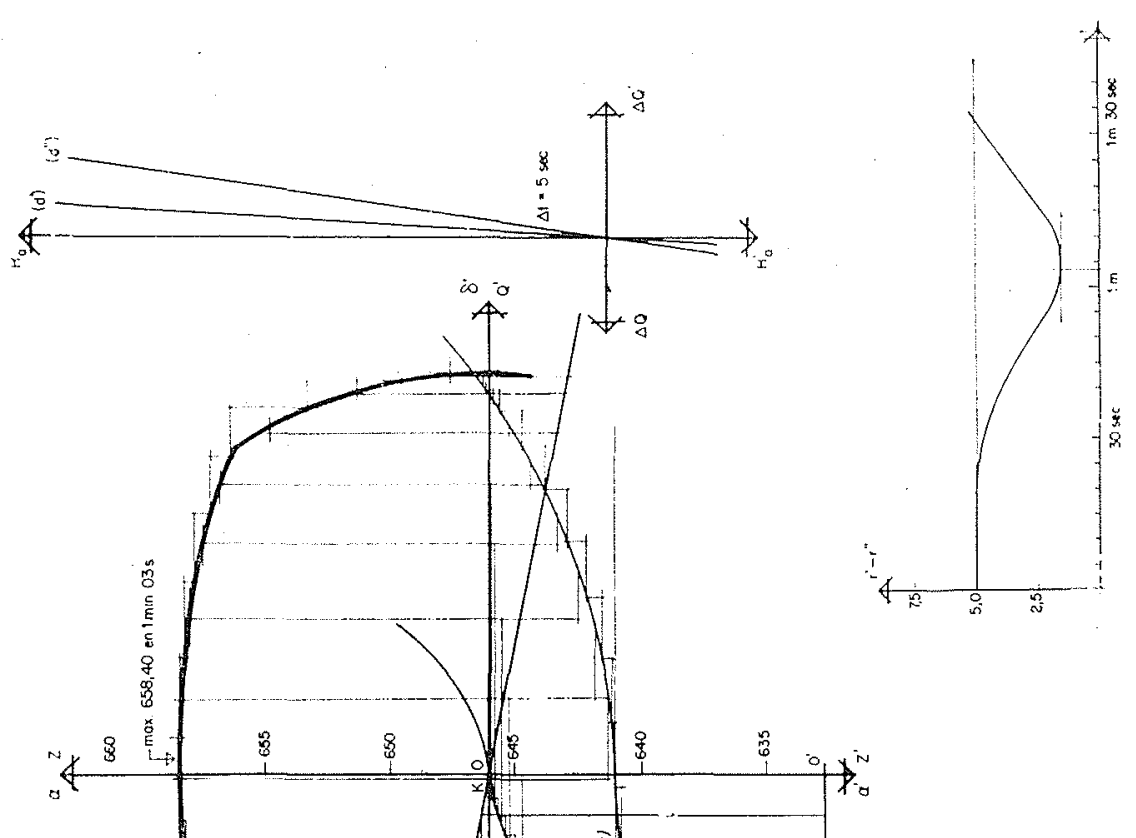


\section{CONCLUSION (1)}

La méthode graphique exposéc, qui a polle origine l'article de Schrrmas cité en référence, nous a paru intéressante a cause de sa grande généralité. En efret :

-... La forme de la cheminée d'équilibre n'intervient que dans la courbe des volumes (courbe 4), de sorte que les cheminces it sections multiples, aussi compliquées soient-elles, se traitent aussi facilement que les cheminées a scction constante.

- L'axe mobile $x x^{\prime}$ permet d'ćtudier des mancuvres d'ouverture ou de fermeture non instantanées (linéaires ou non) presque aussi facilement que des manouvres instantanées.

-..- Dans le cas des galeries en $Y$, le tube piczométrique fictif placé au confluent des trois galeries permel de ramener le probleme au cas simple des galeries uniques.
- L'emploi de coordonnces non réduites el d'intervalles de temps $\Delta t$ fixes a lavantage de permetle is l'opérateur de suive physiquement le phénomène dacećlération. La moindre erreur dans la construction graphique est par suite facilement décelable.

- Enfin, et ceci n'est pas un détail négligeable, cette méthode est rapide. Pour peu que l'opérateur soit entrainé à son manicment, une épure même compliquée peut se faire en deux ou trois heures. Et les résultats sont (nous l'avons vérifié plusieurs fois) aussi précis que ceux obtenus par les épures de MM. Calame el Gaden.

Nous avons plus particulièrement mis en évidence la notion de hauteur accélératrice qui s'est avérée extrèmement pratique. Nous estimons d'ailleur's qu'elle dépasse le cadre de cette méthode et qu'elle représente une grandeur physique permettant de mieux saisio les phénomènes d'acéléralion dans les cheminées d'équilibre.

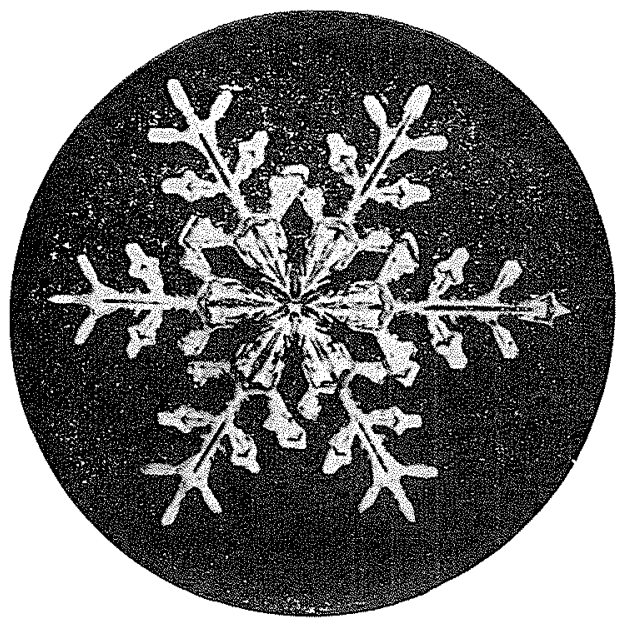

(1) Dans un prochain expose nous ajouterons à cette étude quelques compléments dordre théorique et expérimental. 